\title{
OPEN Feasibility of reintroducing grassland megaherbivores, the greater one-horned rhinoceros, and swamp buffalo within their historic global range
}

\author{
HarshiniY. Jhala ${ }^{1,2 \bowtie}$, Qamar Qureshi ${ }^{2}$, Yadvendradev V. Jhala ${ }^{2}$ \& Simon A. Black ${ }^{1 \bowtie}$
}

Reintroduction of endangered species is an effective and increasingly important conservation strategy once threats have been addressed. The greater one-horned rhinoceros and swamp buffalo have declined through historic hunting and habitat loss. We identify and evaluate available habitat across their historic range (India, Nepal, and Bhutan) for reintroducing viable populations. We used Species Distribution Models in Maxent to identify potential habitats and evaluated model-identified sites through field visits, interviews of wildlife managers, literature, and population-habitat viability analysis. We prioritize sites based on size, quality, protection, management effectiveness, biotic pressures, and potential of conflict with communities. Our results suggest that populations greater than $\mathbf{5 0}$ for rhinoceros and $\mathbf{1 0 0}$ for buffalo were less susceptible to extinction, and could withstand some poaching, especially if supplemented or managed as a metapopulation. We note some reluctance by managers to reintroduce rhinoceros due to high costs associated with subsequent protection. Our analysis subsequently prioritised Corbett and Valmiki, for rhino reintroduction and transboundary complexes of Chitwan-Parsa-Valmiki and Dudhwa-Pilibhit-Shuklaphanta-Bardia for buffalo reintroductions. Establishing new safety-nets and supplementing existing populations of these megaherbivores would ensure their continued survival and harness their beneficial effect on ecosystems and conspecifics like pygmy hog, hispid hare, swamp deer, hog deer, and Bengal florican.

Species are facing an unprecedented extinction crisis due to anthropogenic impacts ${ }^{1}$ with large carnivores and megaherbivores bearing the brunt ${ }^{2}$. These taxa have been extirpated from many of their natural habitats by direct hunting for meat, trophies, crop protection, and retaliatory killing ${ }^{3,4}$. However, large carnivores have been observed to recolonize areas where these threats have been removed, if such areas are connected with source populations ${ }^{5}$. Habitat connectivity for megaherbivores differs to connectivity in carnivores, since carnivores can often pass through degraded habitats, while megaherbivore corridors require forage, water and absence form potential conflict with humans. Megaherbivores share slow life history traits, which make them more vulnerable to threats of habitat loss and poaching ${ }^{6}$. As a consequence, recolonization is rarely observed in megaherbivores other than in elephants ${ }^{7}$, since most source populations are depleted and connecting corridors are degraded or destroyed. Planned reintroductions can be a useful strategy to establish safety-net populations and are often a better option, since natural recolonization is a slow and stochastic process ${ }^{8}$.

Megaherbivore species are largely unaffected by non-human predation, so can sustain high stable densities, allowing them to greatly modify the ecosystems they inhabit ${ }^{9}$. As ecosystem engineers, megaherbivores regulate tall coarse grass through grazing and trampling, which allows the growth of palatable species accessible for consumption by smaller mesoherbivore species ${ }^{10,11}$. Reintroduction and supplementation of megaherbivores would not only create safety-net populations but also restore an important ecological role in currently degraded habitats ${ }^{12}$ and recover the potential of such habitats to sustain historical faunal assemblages.

The Indian subcontinent is home to a diverse range of megaherbivores, including elephant (Elephus maximus), gaur (Bos gaurua), greater one-horned rhinoceros (Rhinoceros unicornis) (hereafter 'rhinoceros'), and

${ }^{1}$ Durrell Institute of Conservation and Ecology, School of Anthropology and Conservation, University of Kent, Canterbury CT2 7NZ, UK. ${ }^{2}$ Wildlife Institute of India, Chandrabani, Dehradun, Uttarakhand 248001, India. ${ }^{\square}$ email: harshiniyj@gmail.com; S.Black@kent.ac.uk 

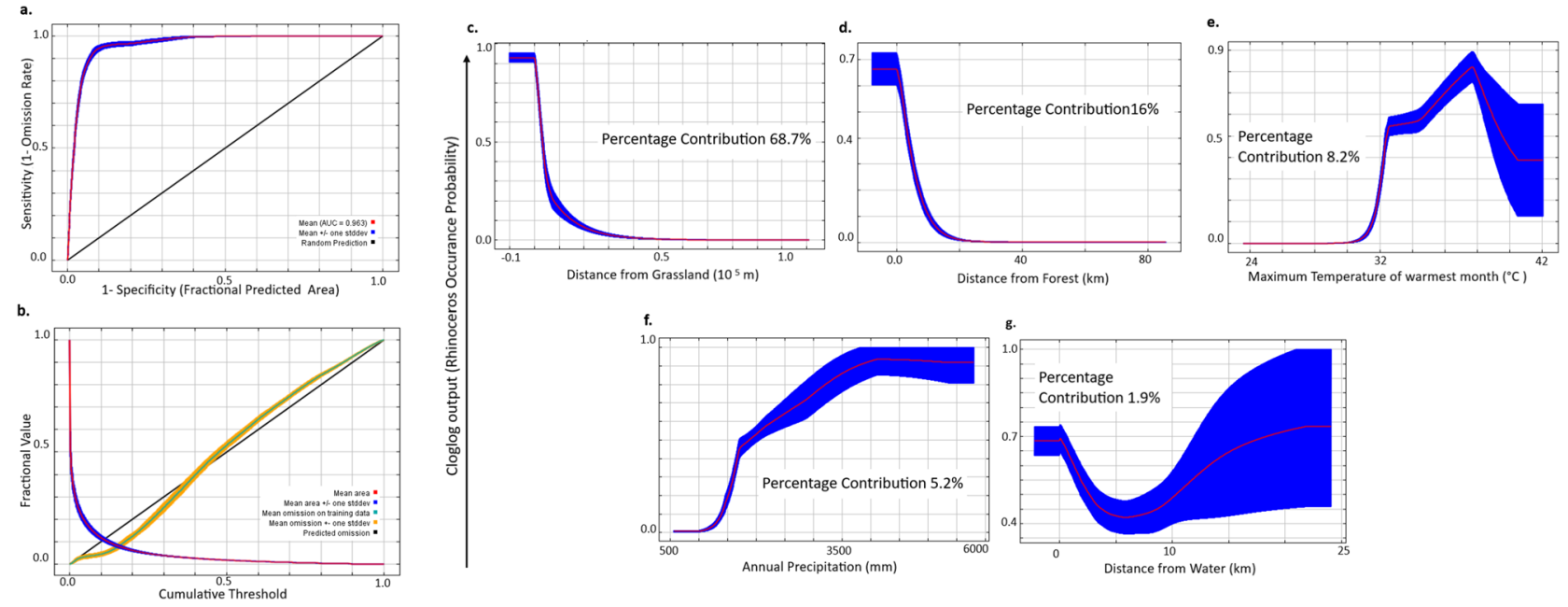

Figure 1. (a) Receiver operator curve for assessing model fit, (b) Omission/Commission analysis for model accuracy of classifying test data. Species occurrence probability (response curves) obtained from 100 bootstrap runs of the best model explaining the distribution of greater one horned rhinoceros in Maxent to (c) distance to grassland, (d) distance to forest, (e) maximum temperature of warmest month, (f) annual precipitation, and (g) distance to water.

wild swamp buffalo (Bubalus arnee) (hereafter 'buffalo'). In recent centuries the populations of rhinoceros and buffalo, both grassland dependent species, have severely declined. Historically, rhinoceros were distributed and abundant across the foothills and floodplains of the Indus (Sindh in Pakistan), Ganges, and the Brahmaputra (up to the Indo-Myanmar border $)^{13}$. Current rhinoceros range is limited and fragmented across India and Nepal as a result of anthropogenic effects ${ }^{14}$. The current global population of rhinoceros is estimated at $\sim 3500$, of which Kaziranga National Park (NP) in Assam, India ( 2400 individuals) and Chitwan NP in Nepal ( 600 individuals) being the only major population strongholds ${ }^{15}$. Similarly, the buffalo was once abundant across the northern and central plains of the subcontinent (from the Indus basin to Brahmaputra floodplains and into south China and Southeast Asia), but is now restricted to small pockets in north-eastern and central India, Nepal, Bhutan, Myanmar, Cambodia and Thailand, with an estimated population of 3000-4000 individuals ${ }^{16}$.

Buffalo and rhinoceros exemplify conservation problems faced by megaherbivores; the rhinoceros more so by being severely persecuted for its horn ${ }^{17}$, whilst the buffalo suffers occasional poaching for bushmeat ${ }^{16}$ and populations of both species have dwindled due to habitat loss. The majority of flood-plain and foot-hill habitat has been converted to agriculture and remaining habitat within Protected Areas (PA) is vulnerable to erosion caused by floods and invasion of exotic plant species like Mikania micantha, Mimosa invisa and Leea crispa ${ }^{14}$. Conflict with people due to economic loss from crop-raiding and occasional human casualties result in negative attitudes in local communities which impedes conservation efforts ${ }^{18}$.

Alongside other criteria, IUCN red listing of species depends on the number and size of populations; with a greater number of large populations endowing higher chances of species survival ${ }^{19}$. Therefore, long-term conservation of megaherbivores depends on establishing viable populations across their global range. This could be achieved through reintroductions into suitable habitats and supplementing existing populations. We use species current occurrence data to model their potential distributions using Maximum Entropy algorithms (Maxent) ${ }^{20}$. These model-identified habitats were evaluated by field surveys, literature review, and discussions with PA managers. We subsequently assessed the feasibility of reintroduction and supplementation based on population habitat viability analysis $\left(\mathrm{PHVA}^{21}\right)$ using parameters of varying levels of poaching, habitat quality, and size. Our study combines information from Species Distribution Models (SDMs), PHVA, management effectiveness, and potential for conflict with humans, in order to prioritize sites for reintroduction and supplementation. We also provide recommendations for enhancing conservation management of extant populations.

\section{Results}

Species distribution modeling. Greater one-horned rhinoceros. Based on Area Under the Curve (AUC) of the Receiver Operator Curve (ROC) and on 100 bootstrap runs of omission/commission analysis with $20 \%$ test data showed that the rhinoceros model had a good fit $[\mathrm{AUC}=0.96$ (SE 0.0007)] and predictive ability (Fig. 1a,b). This model was also rated the best by True Skill Statistics (TSS) and had the lowest Akaike Information Criteria (AIC). Rhinoceros occurrence was best explained by (a) Distance from grassland, (b) Distance from forest, (c) Maximum temperature of warmest month (d) Annual Precipitation and, (e) Distance from water (Table 1). As expected, species response curve showed a decline in habitat suitability with increasing distance from grasslands (Fig. 1c). Rhinoceros were unlikely to occur at distances $>2 \mathrm{~km}$ from grasslands. Distance from grassland habitat explained the maximum variation (69\%) in the rhinoceros distribution model. Rhinoceros occurrence declines rapidly from forest edge (Fig. 1d) which contributed 16\% to the model. A temperature range between 32 to $40{ }^{\circ} \mathrm{C}$ during the warmest months of the year governed occurrence of rhinoceros (Fig. 1e). This covariate (Maximum temperature of the warmest month) explained $8.2 \%$ of the variation in the data in the best model. Rhinoceros 


\begin{tabular}{|c|c|c|c|c|c|c|c|c|c|c|c|}
\hline \multirow[b]{2}{*}{ Variables } & \multicolumn{5}{|l|}{ Rhino } & \multicolumn{5}{|l|}{ Buffalo } & \multirow[b]{2}{*}{ Source } \\
\hline & Training AUC & Test AUC & TSS & Kappa & AIC & Training AUC & Test AUC & TSS & Kappa & AIC & \\
\hline \multicolumn{12}{|l|}{ Climate } \\
\hline $\begin{array}{l}\text { Maximum temperature of warm- } \\
\text { est month (MXT) }\end{array}$ & 0.92 & 0.87 & 0.54 & 0.07 & $10,734.8$ & 0.88 & 0.84 & 0.41 & 0.00 & 1762.0 & Worldclim website \\
\hline Annual precipitation (AP) & 0.89 & 0.85 & 0.69 & 0.05 & $11,501.6$ & 0.85 & 0.82 & 0.35 & 0.00 & 1792.4 & Worldclim website \\
\hline $\begin{array}{l}\text { Minimum temperature of coldest } \\
\text { month (MIT) }\end{array}$ & 0.84 & 0.84 & 0.50 & 0.02 & $11,723.6$ & 0.88 & 0.83 & 0.54 & 0.00 & 1749.4 & Worldclim website \\
\hline $\begin{array}{l}\text { Precipitation of driest quarter } \\
\text { (PQ) }\end{array}$ & 0.88 & 0.85 & 0.53 & 0.03 & $11,356.6$ & 0.82 & 0.75 & 0.19 & 0.00 & 1801.5 & Worldclim website \\
\hline $\begin{array}{l}\text { Precipitation of wettest month } \\
\text { (PW) }\end{array}$ & 0.78 & 0.71 & 0.70 & 0.00 & $12,135.7$ & 0.87 & 0.80 & 0.36 & 0.00 & 1780.5 & Worldclim website \\
\hline \multicolumn{12}{|l|}{ Abiotic habitat } \\
\hline Elevation (E) & 0.87 & 0.82 & 0.40 & 0.01 & $11,654.3$ & 0.87 & 0.82 & 0.42 & 0.00 & 1763.3 & SRTM Data \\
\hline Distance from water (DW) & 0.70 & 0.70 & 0.34 & 0.01 & $12,391.2$ & 0.77 & 0.72 & 0.56 & 0.01 & 1844.9 & $\begin{array}{l}\text { NDWI \& MNDWI } \\
\text { from LandSat } 8\end{array}$ \\
\hline \multicolumn{12}{|l|}{ Biotic habitat } \\
\hline $\begin{array}{l}\text { Distance from grassland (DGL) } \\
\text { and ground validation }\end{array}$ & 0.92 & 0.89 & 0.20 & 0.00 & $10,810.3$ & 0.92 & 0.91 & 0.68 & 0.02 & 1648.9 & $\begin{array}{l}\text { Digitized using Land- } \\
\text { Sat } 8 \text {, Google Earth Pro }\end{array}$ \\
\hline Distance from forest (DF) & 0.78 & 0.79 & 0.40 & 0.01 & $11,954.1$ & 0.73 & 0.72 & 0.29 & 0.00 & 1837.3 & GlobCover (2009) \\
\hline Pre-monsoon NDVI (PRNDVI) & 0.71 & 0.73 & 0.13 & 0.00 & $12,368.2$ & 0.74 & 0.73 & 0.44 & 0.00 & 1897.3 & $\begin{array}{l}\text { MODIS Vegetation } \\
\text { Index Products }\end{array}$ \\
\hline Post-monsoon NDVI (PONDVI) & 0.79 & 0.76 & 0.37 & 0.01 & $12,056.4$ & 0.73 & 0.65 & 0.12 & 0.00 & 1900.6 & $\begin{array}{l}\text { MODIS Vegetation } \\
\text { Index Products }\end{array}$ \\
\hline $\begin{array}{l}\text { Difference in pre-monsoon and } \\
\text { post-monsoon NDVI (DNDVI) }\end{array}$ & 0.70 & 0.63 & 0.15 & 0.00 & $12,397.4$ & 0.66 & 0.62 & 0.06 & 0.00 & 1873.3 & $\begin{array}{l}\text { MODIS Vegetation } \\
\text { Index Products }\end{array}$ \\
\hline \multicolumn{12}{|l|}{ Anthropogenic disturbance } \\
\hline Human footprint Index (HF) & 0.74 & 0.76 & 0.27 & 0.03 & $12,159.0$ & 0.72 & 0.79 & 0.00 & 0.00 & 1844.6 & $\begin{array}{l}\text { Last of the Wild } \\
\text { Project, Version2, } 2005 \\
\text { (LWP-2) }\end{array}$ \\
\hline Distance from PA (DPA) & 0.93 & 0.93 & 0.00 & 0.00 & $10,590.2$ & 0.91 & 0.91 & 0.00 & 0.00 & 1636.7 & $\begin{array}{l}\text { Protected Planet } \\
\text { website }\end{array}$ \\
\hline \multicolumn{12}{|c|}{ Combined climate, biotic and abiotic habitat parameter, and anthropogenic disturbance } \\
\hline $\mathrm{PW}+\mathrm{DGL}+\mathrm{DF}+\mathrm{DW}+\mathrm{PONDVI}$ & 0.95 & 0.95 & 0.77 & 0.09 & $10,376.9$ & 0.97 & 0.98 & 0.82 & 0.02 & 1594.2 & \\
\hline \begin{tabular}{l|l}
$\mathrm{PW}+\mathrm{DGL}+\mathrm{DF}+\mathrm{DW}$ \\
\end{tabular} & 0.95 & 0.94 & 0.79 & 0.10 & $10,581.7$ & 0.96 & 0.99 & 0.850 & 0.016 & 1641.6 & \\
\hline $\mathrm{PQ}+\mathrm{DGL}+\mathrm{DF}+\mathrm{DW}$ & 0.97 & 0.96 & 0.79 & 0.12 & 9620.7 & 0.97 & 0.94 & 0.66 & 0.03 & 1567.2 & \\
\hline $\begin{array}{l}\mathrm{MXT}+\mathrm{AP}+\mathrm{DGL}+\mathrm{DF}+\mathrm{POND} \\
\mathrm{VI}+\mathrm{DW}\end{array}$ & 0.96 & 0.95 & 0.82 & 0.15 & $\underline{10,003.3}$ & $\underline{0.968}$ & 0.958 & 0.75 & 0.045 & 1578.26 & \\
\hline$\underline{M X T}+A P+D G L+D F+D W$ & $\underline{0.97}$ & $\underline{0.96}$ & $\underline{0.84}$ & $\underline{0.11}$ & $\underline{9491.1}$ & 0.96 & 0.96 & 0.68 & 0.04 & 1502.0 & \\
\hline $\mathrm{AP}+\mathrm{DGL}+\mathrm{DF}+\mathrm{DW}$ & 0.97 & 0.95 & 0.80 & 0.12 & 9757.3 & $\underline{0.96}$ & $\underline{0.94}$ & $\underline{0.854}$ & $\underline{0.03}$ & $\underline{1550.4}$ & \\
\hline
\end{tabular}

Table 1. Covariates, their contribution to explaining species occurrence, and their sources. Here AUC- Area Under Curve, TSS- True Skill Statistics and AIC- Akaike Information Criteria. The best models (bold and underlined) were most parsimonious and ecologically meaningful models with high AUC, best TSS and small AIC values.

occurred where annual rainfall exceeded $1700 \mathrm{~mm}$ and the probability of occurrence increased with increase in rainfall up to $4000 \mathrm{~mm}$ (Fig. 1f). Rhinoceros occurrence probability declined sharply with increasing distance to water up to $5 \mathrm{~km}$, after this distance there was high variability in the response curve, This covariate contributed the least to the model at $1.9 \%$ (Fig. $1 \mathrm{~g}$ ).

Swamp buffalo. The Buffalo model had a good fit with an AUC of 0.95 (SE 0.0027) but the predictive ability based on 100 bootstrap runs of omission/commission analysis with $20 \%$ test data (Fig. 2a,b) was not as good as that for rhinoceros. The TSS was the best for this model while the AIC was second best (Table 1). However, since this model had a good fit and made ecological sense supported by TSS we use it as the best model. The covariates of the best buffalo model were (a) Distance from grassland, (b) Distance from forest, (c) Annual Precipitation and, d) Distance from water (Table 1). Distance to grassland had the highest contribution to the model. Buffalo occurred in the proximity of grasslands and were not likely to be found beyond $1 \mathrm{~km}$ distance from grasslands (Fig. 2c). The model showed high buffalo habitat suitability at forest edges with low probability with increasing distances from forests (Fig. 2d). Areas having annual rainfall above $1500 \mathrm{~mm}$ were preferred (Fig. 2e). Habitat suitability for buffalo declined rapidly with increasing distance from water for up to $2 \mathrm{~km}$ after which habitat was unsuitable with high variability in model predictions (Fig. 2f). 

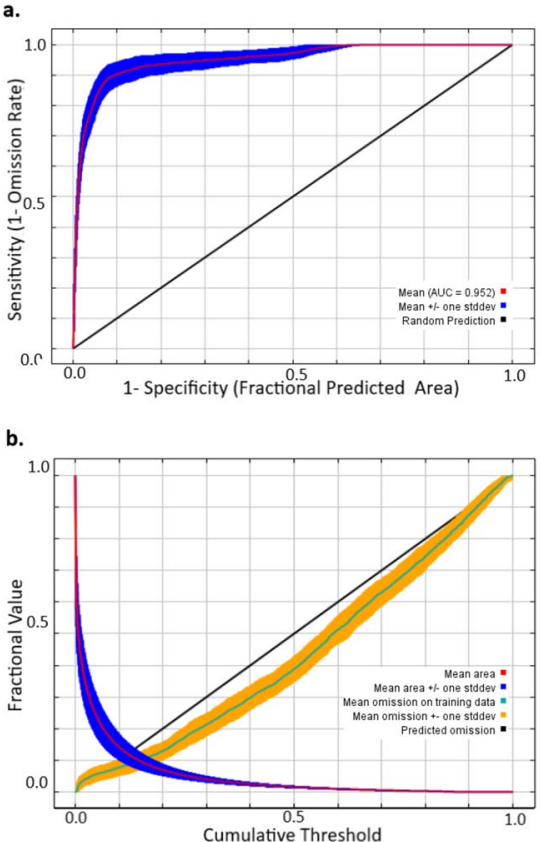
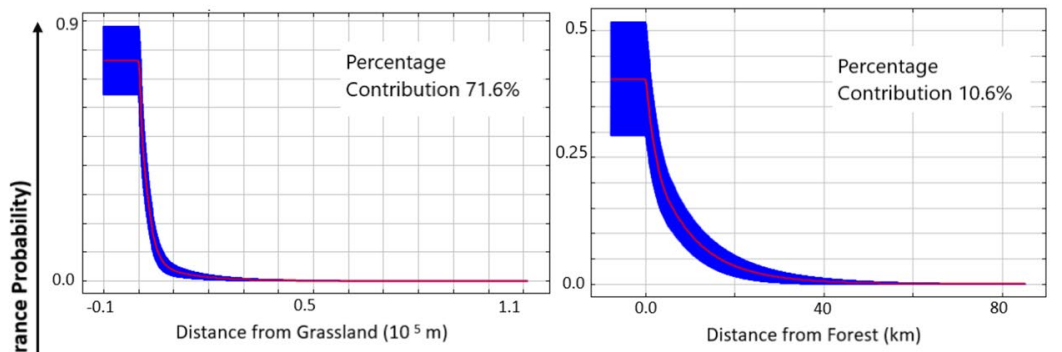

f.

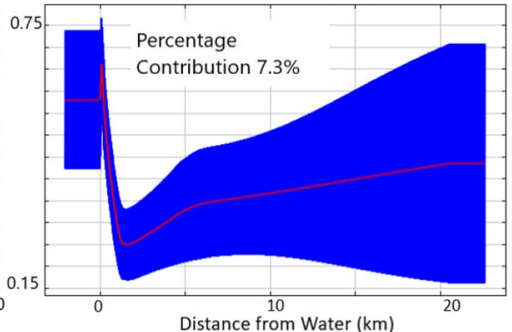

Figure 2. (a) Receiver Operator Curve for assessing model fit, (b) Omission/Commission analysis for model accuracy of classifying test data. Response curves obtained from 100 bootstrap runs of modeling distribution of wild swamp buffalo in Maxent to (c) distance to grassland, (d) distance to forest, (e) Annual precipitation and (f) distance to water for the Terai-Brahmaputra floodplains landscape.

Population habitat viability analysis (PHVA). Greater one-horned rhinoceros. PHVA for rhinoceros revealed small populations ( $\mathrm{K} \leq 10$ ) could not persist (Table 2, Scenarios $1-4$ and Table S1). Medium sized populations $(K=20-30)$ are shown to be viable when initial reintroductions are undertaken with $>8-10$ rhinoceros and sites are occasionally supplemented, but these populations would not withstand poaching (Table 2, Scenarios 5-11 and Table S1,). Populations with $\mathrm{K} \geq 50$ have better chances of survival which increases with supplementation. These populations would also withstand low levels of poaching when supplemented at initial stages (Table 2, Scenarios 14 \& 15). Populations 100 would survive long-term, even when subjected to a low-level poaching, and are able to retain high levels of heterozygosity even without immigrants (Table 2, Scenario 19-21 and Table S1). Populations with $\mathrm{K} \geq 100$ are ideal for long-term persistence (Table 2, Scenario 22). A metapopulation comprising of Kaziranga, Orang and Laokhowa-Bura Chapori demonstrates a stochastic growth rate of 0.022 (SE 0.019). The extinction probability of that metapopulation is zero and heterozygosity is maintained at $~ 100 \%$.

Swamp buffalo. Small populations $(\mathrm{K} \leq 20)$ exhibit low persistence probability despite supplementation (Table 3, Scenarios 1-4 and TableS2). Medium sized populations $(K=50)$ could persist with initial reintroduction of $>10$ individuals supplemented for a decade, but would not sustain poaching offtake (Table 3, Scenarios 5-9 and Table S2). Large populations ( $\mathrm{K}=100-200$ buffalos) remain viable in settings which experience natural catastrophes, and are also resilient to moderate poaching losses if initial founding population is $>30$. Populations are sensitive to the size of founding population and depend upon continued supplementation (Table 3, Scenario 10-16 and Table S2). Areas which could sustain $(\mathrm{K})>250$ were ideal for long-term persistence and could tolerate moderate poaching. However, all populations were sensitive to founding population size, and a founding population $>30$ is optimal.

Identifying and prioritizing suitable habitats. The species distribution probability asc. layer obtained as the median from 100 Maxent bootstrap runs were exported to Arc GIS 10.5 to produce probability maps for rhinoceros (Fig. 3) and buffalo (Fig. 4), showed reasonable extents of suitable habitat outside of these species' current range. Maps from conservative estimates of $95 \%$ lower limits also identified substantial patches of suitable habitat for reintroductions (Fig S1). Maximum training sensitivity plus specificity cumulative threshold values for 100 bootstrap runs for the rhinoceros model was less variable (13.95 \pm SE 0.196; range 9.6-18.5) when compared to that of the buffalo model (19.77 \pm SE 0.88; range 1.7-54.7). Maxent identified 11 and 7 habitat patches for rhinoceros and buffalo respectively that could sustain $>50$ and $>100$ individuals of each species outside of the current range (Table 4). After prioritizing these sites based on legal status, protection, management efficacies and minimal potential for human conflict we identified Corbett NP and Valmiki Tiger Reserve (TR) as top priority sites for rhinoceros reintroduction. For buffalo reintroductions the PA complex of Chitwan NPValmiki TR-Parsa Wildlife Sanctuary (WLS) and Bardia NP-Shuklaphanta NP-Dudhwa NP-Katerniagath WLSPilibhit TR were top priority (Table S3). While, Bardia NP and Shuklaphanta NP in Nepal and Dudhwa NP, Manas NP in India would benefit from Rhinoceros supplementation (Table 4 \& Table S3). 


\begin{tabular}{|c|c|c|c|c|c|c|c|c|c|}
\hline Scenario & Carrying capacity & Initial population & Supplementation & Frequency of catastrophes & Frequency of harvest & $\mathbf{r}(\mathrm{SD})$ & PE & $\mathrm{N}$ & $\mathbf{H} \%$ \\
\hline 1 & 10 & $5(3 \mathrm{AF} \& 2 \mathrm{AM})$ & None & None & None & $0.015(0.126)$ & 0.85 & 5 & 40 \\
\hline 2 & 10 & $5(3 \mathrm{AF} \& 2 \mathrm{AM})$ & None & $4 \%$ flood & None & $0.014(0.127)$ & 0.87 & 5 & 44 \\
\hline 3 & 10 & $5(3 \mathrm{AF} \& 2 \mathrm{AM})$ & $\begin{array}{l}2 \text { in } 2 \text { years }(1 \mathrm{AF} \& 1 \mathrm{AM}) \\
\text { for first } 5 \text { years }\end{array}$ & None & None & $0.029(0.139)$ & 0.84 & 5 & 44 \\
\hline 4 & 10 & $5(3 \mathrm{AF} \& 2 \mathrm{AM})$ & None & $4 \%$ flood & 2 in 5 years $(1 \mathrm{AF} \& 1 \mathrm{AM})$ & $0.021(0.183)$ & 1.00 & 0 & 0 \\
\hline 5 & 20 & $5(3 \mathrm{AF} \& 2 \mathrm{AM})$ & None & None & None & $0.016(0.096)$ & 0.28 & 63 & 12 \\
\hline 6 & 20 & $5(3 \mathrm{AF} \& 2 \mathrm{AM})$ & None & $4 \%$ flood & 2 in 5 years ( $1 \mathrm{AF} \& 1 \mathrm{AM})$ & $0.023(0.176)$ & 1.00 & 0 & 0 \\
\hline 7 & 20 & $8(6 \mathrm{AF} \& 2 \mathrm{AM})$ & $\begin{array}{l}3 \text { in } 2 \text { years }(2 \mathrm{AF} \& 1 \mathrm{AM}) \\
\text { for first } 6 \text { years }\end{array}$ & $4 \%$ flood & 2 in 10 years $(1 \mathrm{AF} \& 1 \mathrm{AM})$ & $0.020(0.109)$ & 25 & 11 & 67 \\
\hline 8 & 20 & $5(3 \mathrm{AF} \& 2 \mathrm{AM})$ & $\begin{array}{l}2 \text { in } 2 \text { years }(1 \mathrm{AF} \& 1 \mathrm{AM}) \\
\text { for first } 5 \text { years }\end{array}$ & $4 \%$ flood & 2 in 5 years $(1 \mathrm{AF} \& 1 \mathrm{AM})$ & $0.003(0.144)$ & 0.96 & 8 & 58 \\
\hline 9 & 20 & $10(7 \mathrm{AF} \& 3 \mathrm{AM})$ & $\begin{array}{l}2 \text { in } 2 \text { years }(1 \mathrm{AF} \& 1 \mathrm{AM}) \\
\text { for first } 5 \text { years }\end{array}$ & $4 \%$ flood & 2 in 5 years ( $1 \mathrm{AF} \& 1 \mathrm{AM})$ & $0.011(0.125)$ & 0.89 & 8 & 63 \\
\hline 10 & 30 & $10(7 \mathrm{AF} \& 3 \mathrm{AM})$ & $\begin{array}{l}3 \text { in } 2 \text { years }(2 \mathrm{AF} \& 1 \mathrm{AM}) \\
\text { for first } 6 \text { years }\end{array}$ & $4 \%$ flood & None & $0.027(0.076)$ & 0.01 & 22 & 77 \\
\hline 11 & 30 & $8(6 \mathrm{AF} \& 2 \mathrm{AM})$ & $\begin{array}{l}3 \text { in } 2 \text { years }(2 \mathrm{AF} \& 1 \mathrm{AM}) \\
\text { for first } 6 \text { years }\end{array}$ & $4 \%$ flood & 2 in 10 years $(1 \mathrm{AF} \& 1 \mathrm{AM})$ & $0.025(0.087)$ & 0.03 & 21 & 78 \\
\hline 12 & 50 & $10(7 \mathrm{AF} \& 3 \mathrm{AM})$ & None & None & None & $0.025(0.06)$ & 0.00 & 41 & 81 \\
\hline 13 & 50 & $10(7 \mathrm{AF} \& 3 \mathrm{AM})$ & $\begin{array}{l}5 \text { (3AF \&2AM) every } \\
2 \text { years for first } 5 \text { years }\end{array}$ & None & None & $0.035(0.078)$ & 0.00 & 42 & 87 \\
\hline 14 & 50 & $10(7 \mathrm{AF} \& 3 \mathrm{AM})$ & $\begin{array}{l}5(3 \mathrm{AF} \& 2 \mathrm{AM}) \text { every } \\
2 \text { years for first } 5 \text { years }\end{array}$ & $4 \%$ flood & 2 in 5 years $(1 \mathrm{AF} \& 1 \mathrm{AM})$ & $0.028(0.84)$ & 0.03 & 35 & 85 \\
\hline 15 & 50 & $10(7 \mathrm{AF} \& 3 \mathrm{AM})$ & $\begin{array}{l}5(3 \mathrm{AF} \& 2 \mathrm{AM}) \text { every } \\
2 \text { years for first } 5 \text { years }\end{array}$ & $4 \%$ flood & 3 in 5 years $(1 \mathrm{AF} \& 2 \mathrm{AM})$ & $0.023(0.087)$ & 0.03 & 35 & 84 \\
\hline 16 & 50 & $10(7 \mathrm{AF} \& 3 \mathrm{AM})$ & $\begin{array}{l}5(3 \mathrm{AF} \& 2 \mathrm{AM}) \text { every } \\
2 \text { years for first } 5 \text { years }\end{array}$ & $4 \%$ flood & 5 in 5 years $(2 \mathrm{AF} \& 3 \mathrm{AM})$ & $0.008(0.119)$ & 0.37 & 26 & 80 \\
\hline 17 & 75 & $10(7 \mathrm{AF} \& 3 \mathrm{AM})$ & None & None & 2 in 5 years ( $1 \mathrm{AF} \& 1 \mathrm{AM})$ & $0.008(0.116)$ & 0.78 & 40 & 75 \\
\hline 18 & 75 & $10(7 \mathrm{AF} \& 3 \mathrm{AM})$ & $\begin{array}{l}5(3 \mathrm{AF} \& 2 \mathrm{AM}) \text { every } \\
2 \text { years for first } 5 \text { years }\end{array}$ & None & 2 in 5 years ( $1 \mathrm{AF} \& 1 \mathrm{AM})$ & $0.029(0.060)$ & 0.00 & 57 & 90 \\
\hline 19 & 100 & $10(7 \mathrm{AF} \& 3 \mathrm{AM})$ & None & None & None & $0.027(0.055)$ & 0.01 & 86 & 84 \\
\hline 20 & 100 & $10(7 \mathrm{AF} \& 3 \mathrm{AM})$ & None & $4 \%$ flood & 2 in 5 years (1AF \&1AM) & $0.008(0.091)$ & 0.39 & 56 & 78 \\
\hline 21 & 100 & $10(7 \mathrm{AF} \& 3 \mathrm{AM})$ & $\begin{array}{l}5(3 \mathrm{AF} \& 2 \mathrm{AM}) \text { every } \\
2 \text { years for first } 5 \text { years }\end{array}$ & $4 \%$ flood & 5 in 5 years $(2 \mathrm{AF} \& 3 \mathrm{AM})$ & $0.020(0.092)$ & 0.12 & 76 & 88 \\
\hline 22 & 150 & $10(7 \mathrm{AF} \& 3 \mathrm{AM}$ & $\begin{array}{l}5 \text { (3AF \&2AM) every } \\
2 \text { years for first } 5 \text { years }\end{array}$ & $4 \%$ flood & 5 in 5 years $(2 \mathrm{AF} \& 3 \mathrm{AM})$ & $0.020(0.090)$ & 0.16 & 108 & 88 \\
\hline
\end{tabular}

Table 2. Results of 500 simulations of population trajectories over 100 years in VORTEX (9.93) to assess the viability of greater one-horned rhinoceros populations with different scenarios of carrying capacity, poaching, catastrophe, initial population size and supplementation. Here AF - adult female, AM - adult male, $r=$ growth rate of population, $(\mathrm{SD})=$ standard deviation, $\mathrm{N}=$ population size at the end of 100 years, $\mathrm{PE}=$ probability of Extinction and $\mathrm{H}=$ heterozygosity of the population at the end of 100 years.

\section{Discussion}

Reintroductions offer great potential for species recovery when following scientifically-informed strategies and relevant risk assessment ${ }^{22}$. Our evaluation of potential reintroduction sites and extant populations addresses these aspects, although any subsequent site-specific plans must also consider IUCN guidelines. Several identified patches sit inside Protected Areas (PAs) with some ready to receive animals, since threats have been addressed and park management capacity exists. We highlight the most feasible actions needed at potential sites. Reintroduction would create safety-net populations for megaherbivores and restore their ecosystem-engineering role, benefitting endangered grassland specialists including pygmy hog (Porcula salvania), hispid hare (Caprolagus hispidus), swamp deer (Rucervus duvaucelii), hog deer (Axis porcinus), and Bengal florican (Houbaropsis bengalensis).

We used a well-established modeling approach of Maxent model ${ }^{23}$ with presence locations obtained from all extant populations to identify potential habitats. Since the modelling space defined in Maxent was clipped within limits of current species' distribution (elevation, temperature, precipitation), the response curves of these variables should be viewed accordingly; we did not model suitable range in Maxent, but rather the high preference habitats within this suitable range. Additionally, covariates used in the final model were uncorrelated, so the response curves of each covariate are similar when compared to individual and cumulative contribution with all other covariates (Fig. S2). By capturing variability in model-based inference on 100 bootstrap runs for 95\% lower limits, inferences remain conservative.

All our presence locations were within PAs (since surviving populations exist only in PAs), so the metric 'distance to PA' would overpredict potential for occurrence in most PAs, we therefore refrained from using this covariate in our final models. Instead we accounted for legal status of habitats after the SDM modelling process during decision-making. Human Footprint Index did not conform to a priori hypothesis and was not used 


\begin{tabular}{|c|c|c|c|c|c|c|c|c|c|}
\hline Scenario & Carrying capacity & Initial population & Supplementation & $\begin{array}{l}\text { Frequency of } \\
\text { catastrophes }\end{array}$ & Frequency of harvest & $\mathbf{r}(\mathrm{SD})$ & PE & $\mathbf{N}$ & $\mathbf{H} \%$ \\
\hline 1 & 20 & $10(6 \mathrm{AF} \& 4 \mathrm{AM})$ & None & None & None & $0.006(0.156)$ & 0.65 & 13 & 34 \\
\hline 2 & 20 & $10(6 \mathrm{AF} \& 4 \mathrm{AM})$ & $\begin{array}{l}2 \text { (1AF \& } 1 \mathrm{AM}) \text { every year } \\
\text { for first } 5 \text { years }\end{array}$ & None & None & $0.013(0.155)$ & 0.62 & 14 & 37 \\
\hline 3 & 20 & $10(6 \mathrm{AF} \& 4 \mathrm{AM})$ & $\begin{array}{l}2 \text { (1AF \&1AM) every year } \\
\text { for first } 10 \text { years }\end{array}$ & $\begin{array}{l}4 \% \text { floods }+2 \% \text { Diseases } \\
\text { outbreak }\end{array}$ & None & $0.015(0.160)$ & 0.67 & 13 & 38 \\
\hline 4 & 20 & $10(6 \mathrm{AF} \& 4 \mathrm{AM})$ & $\begin{array}{l}2 \text { (1AF \& } 1 \text { AM) every year } \\
\text { for first } 10 \text { years }\end{array}$ & None & $\begin{array}{l}2(1 \mathrm{AF} \& 1 \mathrm{AM}) \text { every year } \\
\text { for } 100 \text { years }\end{array}$ & $0.014(0.196)$ & 1 & 0 & 0 \\
\hline 5 & 50 & $10(6 \mathrm{AF} \& 4 \mathrm{AM})$ & None & None & None & $0.014(0.129)$ & 0.43 & 37 & 54 \\
\hline 6 & 50 & $10(6 \mathrm{AF} \& 4 \mathrm{AM})$ & $\begin{array}{l}2 \text { (1AF \& } 1 \mathrm{AM}) \text { every year } \\
\text { for first } 5 \text { years }\end{array}$ & None & None & $0.023(0.111)$ & 0.1 & 39 & 63 \\
\hline 7 & 50 & $10(7 \mathrm{AF}, 3 \mathrm{AM})$ & $\begin{array}{l}3(2 \mathrm{AF} \& 1 \mathrm{AM}) \text { every } \\
2 \text { years for first } 10 \text { years }\end{array}$ & $\begin{array}{l}4 \% \text { floods }+2 \% \text { Diseases } \\
\text { outbreak }\end{array}$ & $\begin{array}{l}2(1 \mathrm{AF} \& 1 \mathrm{AM}) \text { every } \\
15 \text { years for } 100 \text { years }\end{array}$ & $0.020(0.118)$ & 0.11 & 34 & 64 \\
\hline 8 & 50 & $10(6 \mathrm{AF} \& 4 \mathrm{AM})$ & $\begin{array}{l}2 \text { (1AF \& } 1 \text { AM) every year } \\
\text { for first } 10 \text { years }\end{array}$ & $\begin{array}{l}4 \% \text { floods }+2 \% \text { Diseases } \\
\text { outbreak }\end{array}$ & None & $0.022(0.112)$ & 0.06 & 37 & 66 \\
\hline 9 & 50 & $10(6 \mathrm{AF} \& 4 \mathrm{AM})$ & $\begin{array}{l}2 \text { (1AF \&1AM) every year } \\
\text { for first } 10 \text { years }\end{array}$ & None & $\begin{array}{l}2(1 \mathrm{AF} \& 1 \mathrm{AM}) \text { every year } \\
\text { for } 100 \text { years }\end{array}$ & $0.007(0.157)$ & 0.88 & 29 & 58 \\
\hline 10 & 100 & $10(6 \mathrm{AF} \& 4 \mathrm{AM})$ & None & None & None & $0.017(0.125)$ & 0.43 & 78 & 60 \\
\hline 11 & 100 & $20(10 \mathrm{AF} \& 10 \mathrm{AM})$ & None & None & None & $0.021(0.091)$ & 0.05 & 80 & 72 \\
\hline 12 & 100 & $20(10 \mathrm{AF} \& 10 \mathrm{AM})$ & $\begin{array}{l}2 \text { (1AF \& } 1 \text { AM) every year } \\
\text { for first } 5 \text { years }\end{array}$ & None & None & $0.024(0.084)$ & 0.01 & 82 & 77 \\
\hline 13 & 100 & $20(10 \mathrm{AF} \& 10 \mathrm{AM})$ & $\begin{array}{l}2 \text { (1AF \&1AM) every year } \\
\text { for first } 5 \text { years }\end{array}$ & None & $\begin{array}{l}2(1 \mathrm{AF} \& 1 \mathrm{AM}) \text { every } \\
2 \text { years for } 100 \text { years }\end{array}$ & 0.001 & 0.58 & 71 & 71 \\
\hline 14 & 100 & $20(10 \mathrm{AF} \& 10 \mathrm{AM})$ & $\begin{array}{l}2 \text { (1AF \& } 1 \text { AM) every year } \\
\text { for first } 5 \text { years }\end{array}$ & $\begin{array}{l}4 \% \text { floods }+2 \% \text { Diseases } \\
\text { outbreak }\end{array}$ & $\begin{array}{l}2(1 \mathrm{AF} \& 1 \mathrm{AM}) \text { every } \\
2 \text { years for } 100 \text { years }\end{array}$ & $0.013(0.135)$ & 0.76 & 62 & 71 \\
\hline 15 & 100 & $30(20 \mathrm{AF} 10 \mathrm{AM})$ & $\begin{array}{l}2 \text { (1AF \& } 1 \text { AM) every year } \\
\text { for first } 5 \text { years }\end{array}$ & $\begin{array}{l}4 \% \text { floods }+2 \% \text { Diseases } \\
\text { outbreak }\end{array}$ & $\begin{array}{l}2(1 \mathrm{AF} \& 1 \mathrm{AM}) \text { every } \\
2 \text { years for } 100 \text { years }\end{array}$ & $0.001(0.105)$ & 0.36 & 65 & 76 \\
\hline 16 & 200 & $30(20 \mathrm{AF} 10 \mathrm{AM})$ & $\begin{array}{l}2 \text { (1AF \& } 1 \text { AM) every year } \\
\text { for first } 5 \text { years }\end{array}$ & $\begin{array}{l}4 \% \text { floods }+2 \% \text { Diseases } \\
\text { outbreak }\end{array}$ & $\begin{array}{l}2(1 \mathrm{AF} \& 1 \mathrm{AM}) \text { every } \\
2 \text { years for } 100 \text { years }\end{array}$ & $0.008(0.095)$ & 0.27 & 134 & 79 \\
\hline 17 & 250 & $10(10 \mathrm{AF} \& 10 \mathrm{AM})$ & None & $\begin{array}{l}4 \% \text { floods }+2 \% \text { Diseases } \\
\text { outbreak }\end{array}$ & None & $0.020(0.094)$ & 0.08 & 169 & 73 \\
\hline 18 & 250 & 20 (10AF \& 10AM) & None & None & None & $0.025(0.084)$ & 0.05 & 192 & 75 \\
\hline 19 & 250 & $20(10 \mathrm{AF} \& 10 \mathrm{AM})$ & $\begin{array}{l}2 \text { (1AF \&1AM) every } \\
2 \text { years for first } 5 \text { years }\end{array}$ & $\begin{array}{l}4 \% \text { floods }+2 \% \text { Diseases } \\
\text { outbreak }\end{array}$ & $\begin{array}{l}2(1 \mathrm{AF} \& 1 \mathrm{AM}) \text { every } \\
2 \text { years for } 100 \text { years }\end{array}$ & $0.018(0.140)$ & 0.83 & 139 & 71 \\
\hline 20 & 250 & 35 (20AF \& 15AM) & $\begin{array}{l}2(1 \mathrm{AF} \& 1 \mathrm{AM}) \text { every } \\
2 \text { years for first } 10 \text { years }\end{array}$ & $\begin{array}{l}4 \% \text { floods }+2 \% \text { Diseases } \\
\text { outbreak }\end{array}$ & $\begin{array}{l}2(1 \mathrm{AF} \& 1 \mathrm{AM}) \text { every } \\
2 \text { years for } 100 \text { years }\end{array}$ & $0.009(0.091)$ & 0.25 & 161 & 80 \\
\hline 21 & 500 & 20 (10AF \& 10AM) & None & None & None & $0.024(0.089)$ & 0.09 & 307 & 74 \\
\hline 22 & 500 & 35 (20AF \& 15AM) & None & $\begin{array}{l}4 \% \text { floods }+2 \% \text { Diseases } \\
\text { outbreak }\end{array}$ & $\begin{array}{l}2(1 \mathrm{AF} \& 1 \mathrm{AM}) \text { every } \\
2 \text { years for } 100 \text { years }\end{array}$ & $0.001(0.106)$ & 0.48 & 212 & 76 \\
\hline 23 & 500 & 35 (20AF \& 15AM) & $\begin{array}{l}5 \text { ( } 3 \mathrm{AF} \& 2 \mathrm{AM}) \text { every } \\
2 \text { years for first } 5 \text { years }\end{array}$ & $\begin{array}{l}4 \% \text { floods }+2 \% \text { Diseases } \\
\text { outbreak }\end{array}$ & $\begin{array}{l}2(1 \mathrm{AF} \& 1 \mathrm{AM}) \text { every } \\
2 \text { years for } 100 \text { years }\end{array}$ & $0.012(0.088)$ & 0.22 & 254 & 81 \\
\hline
\end{tabular}

Table 3. Results of 500 runs of population habitat viability analysis of wild swamp buffalo populations with different scenarios of carrying capacity, poaching, catastrophe, initial population size and supplementation over a period of 100 years. Here AF - adult female, AM- adult male, $r=$ growth rate of population, $(\mathrm{SD})=$ standard deviation, $\mathrm{N}=$ population size at the end of 100 years, $\mathrm{PE}=$ probability of Extinction and $\mathrm{H}=$ heterozygosity of the population at the end of 100 years.

after exploratory analysis, since most of the fertile floodplain habitats outside the realm of legal protection were already occupied for human land use. Species presence locations were often juxtaposed with pixels having high Human Footprint values due to hard PA boundaries, making it ecologically uninformative. Variables such as 'distance from natural grasslands' and 'distance from forest' better captured negative influences of human impact compared to Human Footprint Index and were aligned with a priori expectations.

Often the test data of species occurrence used to validate models are in close proximity to species presence points used for model building, causing spatial autocorrelation ${ }^{24}$ that inflates the accuracy (AUC) of the model ${ }^{25}$. In both megaherbivore models geographic clustering of training and test data may have caused spatial autocorrelation and inflation of AUC values. However, our assertion is that species occurrence data used in our models spans the entire extant geographic range of both megaherbivores across the entire spectrum of covariate space occupied by each species. We use only one presence location per one $\mathrm{km}^{2}$ pixel (as explained by Philips et al. $2017^{85}$ ), and use the bias correction file option in Maxent to address this limitation of training data ${ }^{26}$. We also use TSS and AIC to evaluate our models.

Our habitat suitability analysis showed PAs such as Kishanpur WLS, Sohagibarwa WLS and Nameri TR had suitable habitat to support $\leq 10$ rhinoceros. Results of PHVA for rhinoceros suggest small populations $(\leq 10)$ had low probability of persistence so these areas are rejected. Suitable habitat identified in Rajaji NP was in the 


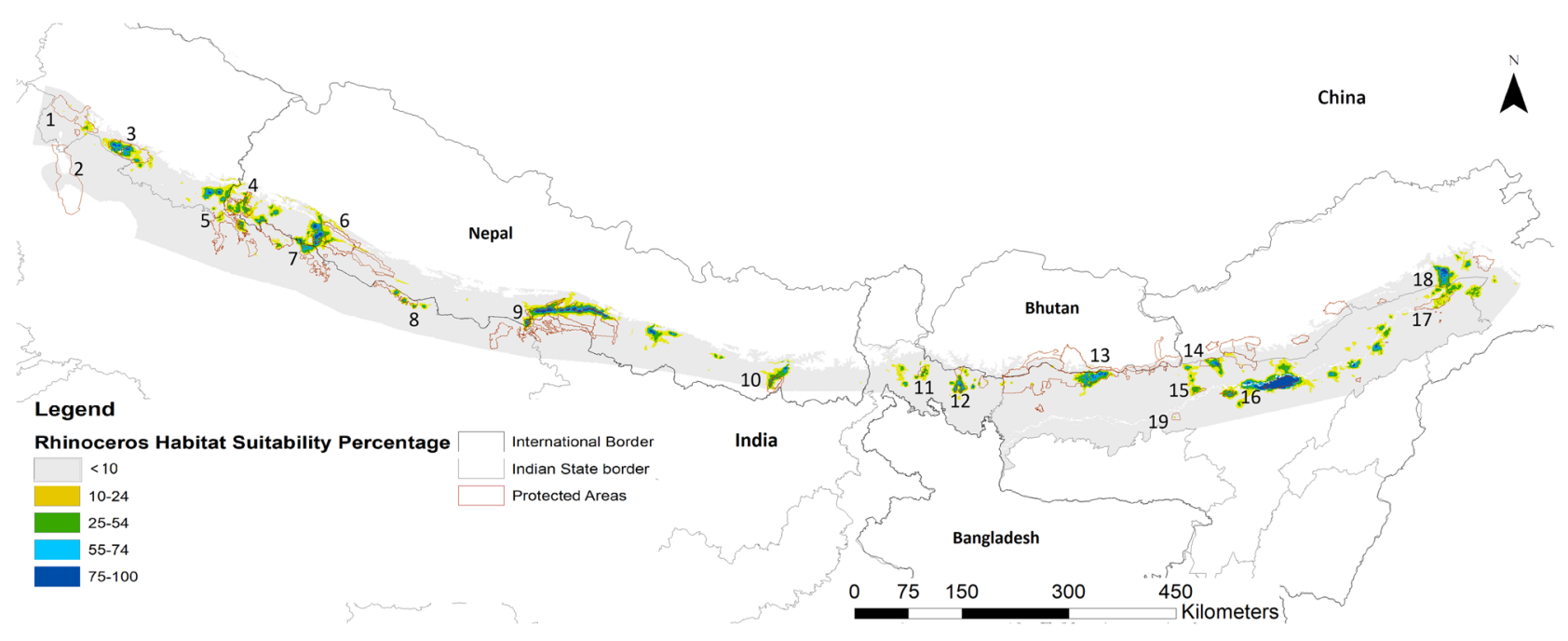

Figure 3. Distribution probability of greater one-horned Rhinoceros across its global historic range in the Terai-Brahmaputra floodplain, where 1-Rajaji NP, 2-Hastinapur WLS 3-Corbett NP, 4- Shukalaphanta NP, 5-Pilibhit TR, 6-Bardia, 7-Keterniyaghat WLS, 8- Sohelwa WLS, 9- Chitwan NP-Valmik TR-Parsa WLS Complex, 10-Koshi Tappu RAMSAR Site, 11- Gorumara WLS, 12- Jaldapara WLS, 13- Manas-Royal NP-Manas NP Complex, 14-Sonai Rupai WLS, 15- Orang TR, 16- Kaziranga NP, 17-Dibru Saikhowa WLS, 18- D’Ering Memorial WLS, 19- Pobitora WLS. Created in ESRI ArcMap 10.5.1 (https://support.esri.com/en/Products/Deskt op/arcgis-desktop/arcmap/10-5-1\#downloads).

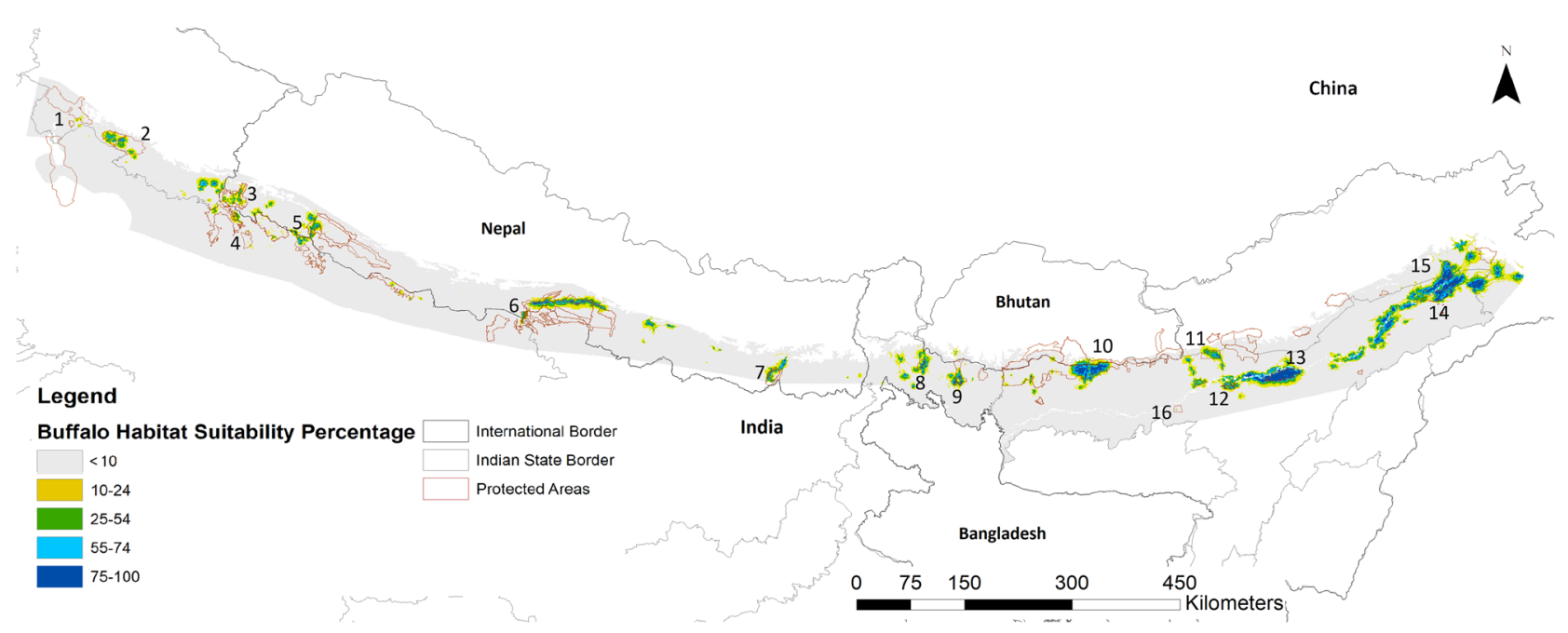

Figure 4. Distribution probability of wild swamp buffalo across the Terai-Brahmaputra floodplain, where 1-Jhilmil Jheel, 2-Corbett NP, 3- Shukalaphanta NP, 4- Kishanpur WLS, 5-Keterniyaghat WLS, 6- Chitwan NP-Valmik TR-Parsa WLS Complex, 7-Koshi Tappu RAMSAR Site, 8- Gorumara WLS, 9- Jaldapara WLS, 10Manas-Royal NP-Manas NP Complex, 11-Sonai Rupai WLS, 12- Laokhowa-Burachapori WLS, 13- Kaziranga NP, 14-Dibru Saikhowa WLS, 15- D’Ering Memorial WLS, 16- Pobitora WLS Created in ESRI ArcMap 10.5.1 (https://support.esri.com/en/Products/Desktop/arcgis-desktop/arcmap/10-5-1\#downloads).

floodplains of the Ganges and its tributaries, an area heavily utilized for religious pilgrimage in proximity to townships of Haridwar and Rishikesh, again unsuitable for reintroduction due to potential human-wildlife conflict.

Although our analysis suggests that Valmiki itself could support only a small population (35-40 individuals), we recommend reintroduction, since Valmiki receives natural immigrants from Chitwan (Nepal), so offers probability of long-term metapopulation persistence. That said, investment to re-route the railway line passing through prime rhinoceros habitat is necessary, and is being considered by the Bihar Government (Valmiki Park Director 2018, Personal Communication). An increase in law enforcement and reduction of human activities are also required, although Valmiki has clearly improved its protection regime in recent years as evidenced by an increasing tiger population ${ }^{27}$, a species vulnerable to poaching ${ }^{28}$.

Encouragingly, existing rhino ranges in Shuklaphanta, Bardia, and Orang have potential to sustain higher numbers. Supplementation would be possible if there is investment in protection, habitat management, and reduction of anthropogenic pressures and human-rhinoceros conflict. The same is needed for existing 


\begin{tabular}{|c|c|c|c|c|c|c|c|c|c|c|c|c|c|c|c|}
\hline \multirow[b]{2}{*}{ Protected area name } & \multirow[b]{2}{*}{ State, country } & \multirow[b]{2}{*}{ Protected status } & \multicolumn{6}{|l|}{ Rhinoceros } & \multicolumn{6}{|l|}{ Buffalo } & \\
\hline & & & $\begin{array}{l}\text { Habitat size } \\
\text { (95\% upper } \\
\text { Cl) } \mathrm{Km}^{2}\end{array}$ & $\begin{array}{l}\text { Habitit size } \\
\text { (median) } \mathrm{km}^{2}\end{array}$ & $\begin{array}{l}\text { Habitat size } \\
(95 \% \text { lower } \\
\text { Cl) } \mathrm{km}^{2}\end{array}$ & $\begin{array}{l}\text { Density (per } \\
\mathrm{km}^{2} \text { ) }\end{array}$ & $\begin{array}{l}\text { Current } \\
\text { population }\end{array}$ & \begin{tabular}{|l|}
$\begin{array}{l}\text { Potential rhino } \\
\text { population } \\
\text { (minimum) }\end{array}$ \\
\end{tabular} & $\begin{array}{l}\text { Habitat size } \\
\text { (95\% upper } \\
\text { Cl) } \mathrm{Km}^{2}\end{array}$ & $\begin{array}{l}\text { Habitat size } \\
\text { (median) } \mathrm{km}^{2}\end{array}$ & $\begin{array}{l}\text { Habitat size } \\
\text { (195\% lower } \\
\text { C1) } \mathrm{km}^{2}\end{array}$ & $\begin{array}{l}\text { Density } \\
\left.\text { (per kmm }{ }^{2}\right)\end{array}$ & Current population & $\begin{array}{l}\begin{array}{l}\text { Potential } \\
\text { population } \\
\text { (minimum) }\end{array} \\
\end{array}$ & \\
\hline $\lim$ (Corbett) & \begin{tabular}{|l|} 
Uttarakhand, \\
India
\end{tabular} & \begin{tabular}{|l} 
National Park, \\
Tiger Reserver
\end{tabular} & 375 & 254 & 177 & 1 & 0 & $254(177)$ & 243 & 134 & 27 & 1 & 0 & 134(27) & \begin{tabular}{|l}
$\begin{array}{l}\text { Isolated } \\
\text { popula- } \\
\text { tion }\end{array}$ \\
ton
\end{tabular} \\
\hline Katerniagath & \begin{tabular}{|l|} 
Uttar Pradesh, \\
India
\end{tabular} & \begin{tabular}{|l|} 
Wildlife \\
Sanctuary
\end{tabular} & 143 & 136 & 116 & 1 & 0 & $136(116)$ & 184 & 167 & 145 & 1 & 0 & $167(145)$ & \begin{tabular}{|l}
$\begin{array}{l}\text { Meta } \\
\text { popula- } \\
\text { tion }\end{array}$ \\
\end{tabular} \\
\hline Plibhit & \begin{tabular}{|l|} 
Uttar Pradesh, \\
India
\end{tabular} & Tiger Reserve & 222 & 124 & 31 & 1 & 0 & 124(31) & 156 & 63 & 3 & 1 & 0 & 63(3) & \\
\hline Dudhwa & \begin{tabular}{|l|} 
Uttar Pradesh, \\
India
\end{tabular} & Tiger Reserve & 172 & 72 & 42 & 1 & $\sim 60$ & $72(42)$ & 171 & 35 & 6 & 1 & 0 & $35(6)$ & \\
\hline Kishanpur & \begin{tabular}{|l|} 
Uttar Pradesh, \\
India
\end{tabular} & \begin{tabular}{|l}
$\begin{array}{l}\text { Wildlife } \\
\text { Sanctuary }\end{array}$ \\
\end{tabular} & 7 & 1 & 0 & 1 & 0 & 1 & 25 & 2 & 0 & 1 & 0 & 2 & \\
\hline Shuklaphanta & Nepal & National Park & 324 & 279 & 145 & 1 & -30 & 279 (145) & 279 & 173 & 32 & 1 & 0 & $173(32)$ & \\
\hline Banke & $\begin{array}{l}\text { Nepal } \\
\end{array}$ & National Park & ${ }_{33}$ & 1 & 0 & 1 & 0 & 1 & 0 & 0 & 0 & 1 & 0 & 0 & \\
\hline Bardia & Nepal & National Park & 387 & 304 & 207 & 1 & -15 & $304(207)$ & 106 & 67 & 54 & 1 & 0 & $67(54)$ & \\
\hline Valmiki & Bihar, India & Tiger Reserve & 52 & 44 & 33 & 1 & $\begin{array}{l}\text { Occasional } \\
\text { immigrants from } \\
\text { Chitwan }\end{array}$ & $44(33)$ & 35 & 27 & 10 & 1 & 0 & $27(10)$ & \begin{tabular}{|l} 
Meta \\
popula- \\
tion
\end{tabular} \\
\hline Chitwan & Nepal & National Park & 731 & 619 & 502 & 1.4 & -600 & $867(703)$ & 689 & 461 & 248 & 1 & $\begin{array}{l}\text { Recently introduced } \\
\text { population }(<20)\end{array}$ & $461(248)$ & \\
\hline Parsa & Nepal & Wildlife Reserve & 43 & 38 & 34 & 1 & 0 & $38(34)$ & 49 & 29 & 17 & 1 & 0 & $29(17)$ & \\
\hline $\begin{array}{l}\text { Laukhowa-Bura } \\
\text { Chapori Complex }\end{array}$ & Assam, India & $\begin{array}{l}\text { Wildifif Sanctu- } \\
\text { ary complex }\end{array}$ & 95 & 95 & 75 & 1 & 1 & $95(75)$ & 97 & 95 & 58 & 1 & 0 & $95(58)$ & \begin{tabular}{|l}
$\begin{array}{l}\text { Meta } \\
\text { popula- } \\
\text { tion }\end{array}$ \\
\end{tabular} \\
\hline Orang & Assam, India & Tiger Reserve & 63 & 51 & 28 & 2 & -30 & $102(56)$ & 65 & 61 & 27 & 1 & 0 & 61 (27) & \\
\hline Kaziranga & \begin{tabular}{|l|l|} 
Assam, India \\
\end{tabular} & National Park & 368 & 368 & 368 & $6^{a}$ & -2400 & 2208 & 368 & 368 & 367 & $4.2^{a}$ & \begin{tabular}{|l|l|}
$\sim 600$ \\
\end{tabular} & 1546 & \\
\hline Gorumara & \begin{tabular}{|l|} 
West Bengal, \\
India
\end{tabular} & National Park & 43 & 33 & 25 & $0.6^{a}(2)$ & $\sim 50$ & $66(50)$ & 70 & 51 & 28 & 1 & 0 & $51(28)$ & \begin{tabular}{|l}
$\begin{array}{l}\text { Isolated } \\
\text { popula- } \\
\text { tion }\end{array}$ \\
\end{tabular} \\
\hline Jaldapara & $\begin{array}{l}\text { West Bengal, } \\
\text { India }\end{array}$ & \begin{tabular}{|l|} 
Willifie \\
Sanctuary
\end{tabular} & 155 & 134 & 122 & $1.7 a$ & -230 & $227(207)$ & 145 & 103 & 76 & 1 & 0 & $103(76)$ & \begin{tabular}{|l|}
$\begin{array}{l}\text { Isolated } \\
\text { popula- } \\
\text { tion }\end{array}$ \\
\end{tabular} \\
\hline Koshi Tappu & Nepal & $\begin{array}{l}\text { Willifie Reserve, } \\
\text { RAMSAR Site }\end{array}$ & 86 & 76 & 50 & 1 & 0 & $76(50)$ & 124 & 85 & 34 & $1.4^{a}$ & $\sim 250$ & 250 & \begin{tabular}{|l}
$\begin{array}{l}\text { Isolated } \\
\text { popula- } \\
\text { tion }\end{array}$ \\
\end{tabular} \\
\hline Royal Manas & Bhutan & National Park & 26 & 17 & 8 & 1 & 0 & $17(8)$ & 216 & 138 & 56 & 1 & & $138(56)$ & \begin{tabular}{|l}
$\begin{array}{l}\text { Meta } \\
\text { popula- } \\
\text { tion }\end{array}$ \\
\end{tabular} \\
\hline Manas & \begin{tabular}{|l|l|} 
Assam, India \\
\end{tabular} & \begin{tabular}{|l|l|l|l} 
National Park \\
\end{tabular} & 604 & 532 & 448 & 1 & -30 & $532(448)$ & 901 & 670 & 537 & 1 & $>500$ & $670(537)$ & \\
\hline $\begin{array}{l}\text { D'Ering Memorial } \\
\text { (Lali) }\end{array}$ & \begin{tabular}{|l|} 
Arunachal \\
Pradesh, India
\end{tabular} & $\begin{array}{l}\text { Widlife } \\
\text { Sanctuary }\end{array}$ & 196 & 194 & 192 & 1 & 0 & 192 & 196 & 195 & 186 & 1 & Unknown $<100$ & $195(186)$ & \begin{tabular}{|l}
$\begin{array}{l}\text { Meta } \\
\text { popula- } \\
\text { tion }\end{array}$ \\
\end{tabular} \\
\hline Dibru Saikhowa & Assam, India & \begin{tabular}{|l|l|l|l} 
National Park \\
\end{tabular} & 221 & 169 & 84 & 1 & 0 & $169(84)$ & 361 & 328 & 270 & 1 & $>100$ & 328 (270) & \\
\hline Sonai Rupai & Assam, India & \begin{tabular}{|l|} 
Widlifie \\
Sanctuary
\end{tabular} & 154 & 139 & 127 & 1 & 0 & $139(127)$ & 168 & 143 & 97 & 1 & 0 & $143(97)$ & \begin{tabular}{|l|}
$\begin{array}{l}\text { Isolated } \\
\text { popula- } \\
\text { tion }\end{array}$ \\
\end{tabular} \\
\hline Buxa & \begin{tabular}{|l} 
West Bengal, \\
India
\end{tabular} & \begin{tabular}{|l|} 
Tiger Reserve \\
\end{tabular} & 21 & 10 & 4 & 1 & 0 & $10(4)$ & 8 & 0 & 0 & 1 & $<20$ & & \begin{tabular}{|l}
$\begin{array}{l}\text { Isolated } \\
\text { popula- } \\
\text { tion }\end{array}$ \\
\end{tabular} \\
\hline Rajaji & \begin{tabular}{|l|} 
Uttrakhand, \\
India
\end{tabular} & National Park & 171 & 76 & 34 & 1 & 0 & $876(34)$ & & & & & 0 & & \begin{tabular}{|l}
$\begin{array}{l}\text { Isolated } \\
\text { popula- } \\
\text { tion }\end{array}$ \\
ton
\end{tabular} \\
\hline Sohagibarwa & \begin{tabular}{|l|} 
Uttar Pradesh, \\
India
\end{tabular} & $\begin{array}{l}\text { Willife } \\
\text { Sanctuary }\end{array}$ & 12 & 6 & 0 & & 0 & 0 & 19 & 5 & 0 & 1 & 0 & 5 & \begin{tabular}{|l}
$\begin{array}{l}\text { Isolated } \\
\text { popula- } \\
\text { tion }\end{array}$ \\
\end{tabular} \\
\hline Sohelva & \begin{tabular}{|l|} 
Uttar Pradesh, \\
India
\end{tabular} & \begin{tabular}{|l} 
Willifie \\
Sanctuary
\end{tabular} & 165 & 109 & 74 & 1 & & $109(74)$ & 87 & 26 & 3 & 1 & 0 & $26(3)$ & \begin{tabular}{|l|}
$\begin{array}{l}\text { Isolated } \\
\text { popula- } \\
\text { tion }\end{array}$ \\
\end{tabular} \\
\hline Nameri & Assam, India & Tiger Reserve & 72 & 12 & 0 & 1 & & 12 & 6 & 0 & 0 & & 0 & 0 & \begin{tabular}{|l|}
$\begin{array}{l}\text { Isolated } \\
\text { popula- } \\
\text { tion }\end{array}$ \\
\end{tabular} \\
\hline Gibbon & Assam, India & \begin{tabular}{|l|} 
willifie \\
Sanctuary
\end{tabular} & 2 & & & & & 0 & & & & & 0 & 0 & \begin{tabular}{|l} 
Isolated \\
popula- \\
tion
\end{tabular} \\
\hline Sonanadi & \begin{tabular}{|l|} 
Uttarakhand, \\
Indiaa
\end{tabular} & \begin{tabular}{|l|}
$\begin{array}{l}\text { Wildifie } \\
\text { Sanctuary }\end{array}$ \\
\end{tabular} & 254 & 233 & 207 & 1 & & $233(208)$ & 246 & 170 & 67 & 1 & 0 & $170(67)$ & \begin{tabular}{|l}
$\begin{array}{l}\text { Isolated } \\
\text { popula- } \\
\text { tion }\end{array}$ \\
ton
\end{tabular} \\
\hline Jhilmil heel & \begin{tabular}{|l|} 
Uttarakhand, \\
India
\end{tabular} & \begin{tabular}{|l|} 
Conservation \\
Reserve
\end{tabular} & & & & 1 & & 0 & 3 & 0 & 0 & & 0 & 0 & $\begin{array}{l}\text { Isolated } \\
\text { popula- } \\
\text { tion }\end{array}$ \\
\hline Pobitora* & Assam, India & $\begin{array}{l}\text { Wildifie } \\
\text { Sanctuary }\end{array}$ & 7 & 4 & 3 & $2.6^{\circ}$ & $\sim 100$ & ${ }^{* 102}$ & 6 & 4 & 2 & $2.6^{\mathrm{a}}$ & $\sim 100$ & ${ }^{100}$ & $\begin{array}{l}\text { Isolated } \\
\text { popula- } \\
\text { tion }\end{array}$ \\
\hline
\end{tabular}

Table 4. Suitable habitats for rhinoceros and buffalo, their legal status, current/potential population that each site can sustain and possibility of metapopulation structure. ${ }^{*}$ Pobitora has extant population of $\sim 100$ Greater one-horned Rhinoceros and 100 Buffalos. a Actual know crude density at extant sites.

reintroduced populations in Dudhwa and Manas which would also benefit from supplementation to mitigate inbreeding ${ }^{29,30}$. Manas NP could support around 500 rhinoceros, but the current population is restricted to low densities in the eastern part of the PA. Manas park managers were reluctant to increase rhinoceros numbers due to a lack of infrastructure to ensure protection (Manas Park Director 2018, Personal Communication). Concerns 
are well-founded; the region suffers socio-political unrest and Manas previously lost rhinoceros to poaching during civil unrest in the 1990 ' $\mathrm{s}^{18}$. Significant investment in training, vehicles, weapons, and M-STrIPES patrolling tool is required for recovery of rhinoceros (and tiger) numbers in Manas NP. Royal Manas NP (Bhutan) is contiguous with Indian Manas NP and could independently support 20 rhinoceros, but managers have similar concerns about resources to protect reintroduced animals (Royal Manas Park Director 2018, Personal Communication). Social instability makes translocation to Manas or Royal Manas difficult.

Our analysis suggests that Corbett TR can sustain a population of $>150$ rhinoceros (Table 4). Site evaluation and habitat studies (including grassland food-source species like Saccrum, Imparata, Arundo and Vetivaria) ${ }^{31}$ suggest that Corbett is suitable, with ample surface water (rivers, reservoirs, pools) ${ }^{32}$ plus sufficient grassland patches interspersed with forest cover, to support mixed foraging and cover during calving and in winters ${ }^{33}$. Habitat management such as artificial wallows may be required, since the largely Bhabhar landscape lacks muddy swampland and oxbow lakes of the Terai. The level of protection in the park is high with managers capable of handling reintroduction ${ }^{34}$. A key benefit of Corbett over other PAs is political stability in Uttarakhand, including in and around the PA (in contrast to Assam and $\mathrm{Nepal}^{18}$ ). The large valleys of Corbett TR offer ideal habitats for rhinoceros, bounded by the lower Himalayas (north), Shivalik Hills (south) and the Ramganga Reservoir, retaining rhinoceros within the $\mathrm{PA}$, so limiting conflict.

Laukhowa-Bura-Chapori complex, proposed for reintroductions by Rhino Vision $2020^{36}$, was also identified by our study, however considerable conservation investments are needed before reintroduction (Fig. S3). Major interventions needed include (a) reduction of cattle grazing, (b) grassland management and weed control, (c) protection (guards, weapons, law enforcement training, vehicles, and infrastructure). The site is of particularly significance ${ }^{27}$, as it connects habitat along the Brahmaputra river for rhinoceros, elephants, buffalo and tigers dispersing from Kaziranga. Our PHVA analysis shows that if rhinoceros in Orang TR, Kaziranga NP and Laokhowa-Bura Chapori WLS complex are managed as a metapopulation they will persist even in the small reserves of Orang and Laokhowa-Bura Chapori for the long-term. Although Dibru Saikhowa WLS (Rhino Vision 2020 site $)^{36}$ and D'Ering offer extensive habitat, both are affected by seasonal agriculture encroachment and high livestock density. Effort to remove or reduce those threats would enable these island sanctuaries to hold an estimated $>100$ rhinoceros each.

One potential metapopulation includes the complex of Bardia and Shuklaphanta of Nepal across the international border to the Indian complex of Dudhwa, Katerniagath, and Pilibhit along the Khata Corridor, LaggaBagga WLS and flood-plains of Sharda river ${ }^{37}$. Rhinoceros sometimes transit these PAs, however new proposed border roads will create further obstacles to transboundary wildlife movements. Metapopulation connectivity would benefit other species like (tigers, elephants, buffalos), reducing the need for supplementation.

Corbett is isolated with no adjacent rhinoceros or buffalo populations, however its large carrying capacity would make a population viable if genetically diverse founders are selected, supported by supplementation. Hastinapur WLS in Uttar Pradesh ${ }^{38}$ and Surai Range in Uttarakhand were previously considered for rhinoceros, but our analysis rejects both. Hastinapur WLS lacks forest cover (essential for thermoregulation and calving) and is bordered by agriculture and high human density. The Surai range, although Terai habitat, is small and suffers human encroachment.

We have modelled supplementation for a period of 5-10 years, a time period coinciding with most government funded projects. If however, supplementation continues intermittently over the long-term it would dramatically improve persistence and genetic variability of populations. Our population estimates from model-based computations for Chitwan and Kaziranga match the current estimates of rhinoceros at these sites ${ }^{15}$ which suggests that extant populations are nearing carrying capacity and can serve as source populations ${ }^{39}$. Pobitora WLS, which has close to 100 rhinoceros and a sizable buffalo population, was not identified as good habitat in our results. This shows the limitations of model-driven inferences, which may fail to account for outliers resulting from species' behavioral plasticity or exceptional tolerance by local people. Pobitora is unique, in being imbedded in a human dominated landscape with minimal availability of optimal conditions, yet rhinoceros and buffalo persist in this remnant $\left(39 \mathrm{~km}^{2}\right)$ due to community tolerance (Fig. S5).

Our results show (somewhat unexpectedly considering illegal demand for rhinoceros horn) that buffalo are more prone to extinction than rhinoceros, which is actually congruent with the IUCN Red List ${ }^{40}$ which categorises the rhinoceros as vulnerable and buffalo as endangered. Buffalo are rarely poached commercially, but our results suggest that even small off-take due to bush-meat demands would be a major cause of concern. Our PHVA results for buffalo are likely conservative since studies on demographic parameters of wild buffalo are sparse and potentially biased towards higher mortality estimates.

Many buffalo populations of Northeast India are either too small or possibly hybridized with domestic buffalo (derived from swamp buffalo ${ }^{16}$. In western regions buffalo are largely locally extinct including in locations which could potentially sustain populations. It would be prudent to reintroduce Bubalus arnee into suitable western sites, since domestic buffalo in those localities are derived from river buffalos (Bubalus bubalus $)^{41,42}$, presenting less risk of hybridization.

Priority sites for buffalo reintroduction in the subcontinent are Chitwan NP-Valmiki TR complex and Shuklaphanta-Bardia-Dudhwa-Katerniagath-Pilibhit complex since both could sustain $>400$ individuals. PAs like Sonai Rupai, D’Ering, Dibru Saikhowa, Jaldapara, and Royal Manas could each hold more than 100 individuals, with the potential to be managed as metapopulations. However, bushmeat hunting persists in northeastern India ${ }^{43}$, which poses a major threat to wild buffalo. Higher densities of buffalo may not be possible until human pressures are reduced and management of invasive plants is undertaken. The model-inferred median population of buffalo sustainable in Corbett NP is about 130 individuals. However, there was high level of uncertainty in our model-based estimates for Corbett (Table 4), which we believe was due to the sampling of presence locations being limited to extant populations in the far east region, which experiences different bioclimatic conditions to Corbett in the western Terai. 
The reintroduction of buffalo could act as a test-bed for rhinoceros reintroductions, enabling establishment of management protocols, enhancing staff experience and ensuring effective future operations for rhinoceros. Reintroduction of buffalo to Dudhwa should be feasible since park managers already have protection and monitoring systems in place following experience of managing reintroduced rhinoceros. Chitwan NP in ChitwanParsa Complex of Nepal (a high potential site for buffalo reintroduction in this study) has seen recent buffalo reintroduction, which has a good chance of establishment due to habitat quality, local people's awareness of ecosystem services, experience of megaherbivores and economic benefits from wildlife tourism ${ }^{44}$.

Knowledge of population genetics must inform any reintroduction. For buffalos, hybridization with domestic buffalo is a key factor, and animals of pure wild lineage are needed as founders for reintroduced populations. Possibly the last pure representatives of Bubalus arnee are around 50 buffalo surviving in central India (UdantiSitanadi TR, Indravati, in Chhattisgarh and Sironcha in Maharashtra $)^{45,46}$. According to our PHVA models, this Central Indian population suffers a high probability of extinction. The animals reside in a zone of high political unrest, making local conservation difficult ${ }^{47}$. Efforts to rescue these buffalo is vital, including taking animals into captivity for conservation breeding, or translocation.

An important aspect highlighted by our SDM was that both the greater one-horned rhinoceros and the wild swamp buffalo have very narrow tolerance of temperature and precipitation (Fig. 1e,f; S5). This has serious implications for persistence of populations under climate change. Current populations of both species are limited to PAs largely surrounded by human land-use, making natural range-shifts unlikely ${ }^{48}$, so conservation policy and strategy must address this limitation by managing metapopulations. Western (Corbett NP) and central terai (Dudhwa-Katerniagath-Bardia) are at higher elevations compared to the Brahmaputra plains and could provide refuges in the advent of climate change.

Several factors limit the potential for reintroducing species in their historic range, including loss and degradation of habitat and competition with human interests. For rhinoceros, even in areas with good habitat and least potential for conflict with humans, managers were reluctant to reintroduce the species, due to resource demands and likely media and political pressure in the face of potential losses of animals. Suitable habitats and management are insufficient for protecting species with high value in illegal wildlife markets; only changes in human perceptions, values, and strict law enforcement will address illegal demand ${ }^{49}$.

We advocate the establishment of safety-net populations across a varied geographical range, and our recommendations address the uncertainties associated with climate change. Continued anthropogenic pressures mean that maintaining effective ecosystems will require balanced species assemblages. Reintroduction and supplementation of viable populations of keystone species are important tools for sustaining and enhancing vital functions in critical natural systems.

\section{Materials and method}

Field sampling. Field sampling was undertaken to obtain extant rhinoceros and buffalo locations (from India, Nepal and Bhutan) for modelling habitat suitability and assess the reintroduction potential of identified sites. The current extant and potential reintroduction sites were assessed for level of protection, anthropogenic pressures, size, and quality of available habitat for the target species through site visits and interviews with wildlife managers. The interviews addressed managers' perception of management strengths and weaknesses, and attitude towards reintroduction/supplementation of both megaherbivores (Table S3). All interviews were conducted after obtaining informed consent of the respondents. Prior ethical approval for the study was granted by the School of Anthropology and Conservation, University of Kent, UK, (Reference ID-48-PGT-17/18) following the standards of the American Anthropological Association. All research was conducted under the laws, rules, and regulations of each country. Interviews were conducted in English since all wildlife managers interviewed were conversant with English. Information from site visits and interviews were used to evaluate sites that were selected by SDMs for practicality of reintroductions and potential establishment of populations.

Species distribution modelling. Several approaches to modelling SDM are available for data typ used in this study i.e. Presence vs. Background points. These include like (1) Ecological niche factor analysis (ENFA) ${ }^{50}$, (2) Genetic algorithm for Rule-set prediction $(G A R P)^{51}$ (3) Maximum Entropy models (Maxent) ${ }^{53}$. Earlier researchers have compared the performance of these approaches and found that Maxent models outperform the other approaches ${ }^{52}$. We used Maximum Entropy Species Distribution Modelling in Maxent (Version 3.4.1) ${ }^{53,54}$ for modelling potential habitat of rhinoceros and buffalo. Maxent uses machine learning to develop relationships from known species occurrence and background data with ecologically meaningful spatial environmental covariates $^{54}$. The program subsequently predicts potential distribution of species from covariate relationships across modelled space $^{53}$.

Species occurrence data. Species occurrence information from extant populations of both species was obtained by direct sightings with coordinates recorded by hand-held GPS, camera trap photo-capture locations across the Terai-Brahmaputra floodplains in India ${ }^{27}$, and from locations of satellite-GPS-radio-collared rhinoceros in Chitwan $\mathrm{NP}^{14}$. To avoid spatial autocorrelation and oversampling, we picked only one location from an area of $1 \mathrm{~km}^{255}$. A total of 358 rhinoceros locations and 78 buffalo locations were used for Maxent modelling. Historical records of occurrence were not used since many covariates used in our models have changed since historical times. Furthermore, our modelling objective was to identify extant available habitats and not historical species range.

Eco-geographical variables. The following covariates were used as predictive variables for SDM: Distance from grassland 
Grasslands of the study area were digitized using unsupervised and supervised classification ${ }^{56}$ in ESRI ArcMap 10.5.1 (Environmental System Research Institute 2016) using LandSat 8 imagery ${ }^{57}$ and corrected using Google Earth. We ground-validated grassland locations by obtaining coordinates from a hand-held GPS device (Fig S6). Of the 141 ground validation points 125 were correctly classified resulting in an accuracy of $88.6 \%$. Euclidean Distance tool in ArcMap 10.5.1 was used to calculate the distance of each pixel from grassland polygons at a resolution of $30 \mathrm{~m}$. Both rhinoceros and buffalo can be considered as grassland dependent species ${ }^{46,58}$. A priori we expected both rhinoceros and buffalo occurrence probability to be high within, and on the edge of, grassland habitats and to decline rapidly with increasing distance to grasslands.

Distance from forest

Data on forest cover was obtained from GlobCover (2009) at a spatial resolution of $300 \mathrm{~m}^{59}$. Euclidean Distance of each pixel to a forest patch was computed using ESRI ArcMap (10.5.1). Both study species inhabit grassland-forest mosaic habitats ${ }^{46,58}$. We expected both species to have high occurrence in forest-edge habitat with a declining response for increased distance to forests.

Distance from water

Spectral indicators such as Normalized Difference Water Index and Modified Normalized Difference Water index were used to classify waterbodies $\left(>30 \mathrm{~m}^{2}\right)$ from LandSat 8 imagery ${ }^{60}$. However, smaller water bodies which are also used by these species could not be detected by satellite imagery and therefore not accounted for in our models. Euclidean Distance to water for each pixel was calculated using ArcMap 10.5.1. Both rhinoceros and buffalo need ample access to surface water and spend a lot of their time wallowing for thermoregulation ${ }^{61}$. In Subedi's ${ }^{33}$ study all radio-collared rhinoceros' locations in Chitwan NP were within $1.8 \mathrm{~km}$ distance from water bodies. An essential element of rhinoceros and buffalo habitats are flowing rivers, pools and oxbow lakes. Studies on behaviour of both species show the use of pools and oxbow lakes for wallowing especially in the dry season $^{45,62}$. Our expectation was high occurrence in proximity to water with a rapid decline in species occurrence with increasing distance from surface water.

Normalised Difference Vegetation Index (NDVI)

NDVI composites were derived from Moderate Resolution Imaging Spectroradiometer (MODIS) data, which was obtained from online data pool, developed by NASA Land Processes Distributed Active Archive Centres. MODIS products are available at spatial resolution of $250 \mathrm{~m}$ with 16-day interval cycle. Use of NDVI for species modelling enable better predictions when used alongside other ecological variables ${ }^{63}$. In this study, three values of NDVI were used (1) Pre-Monsoon NDVI (March-April), which indexes canopy cover of the driest months, (2) Post-Monsoon NDVI (October-November) which characterise maximum cover, and (3) Difference in NDVI of Post-Monsoon and Pre-Monsoon which indicates monsoonal flush of annuals compared to more perennial canopy cover. We expected rhinoceros and buffalo to have a positive response in occurrence probability to higher pre-monsoon NDVI as these pixels would be correlated with forage availability in resource lean summers. Species occurrence response to NDVI difference was expected to increase with increasing difference, as higher difference to reflects fresh flush of nutritive forage $e^{64,65}$.

Geo-climatic data

Climatic data are commonly used for modelling species distribution ${ }^{60,61,66}$. Both species prefer wetter habitats and moderate climate ${ }^{33,46}$. The modelling extent was clipped by the limits of species occurrence recorded for elevation, temperature and rainfall ${ }^{67}$. Data on (i) annual rainfall (b15, layer clipped for annual rainfall above $1000 \mathrm{~mm}$ ), (ii) precipitation of the driest quarter (b17), (iii) maximum temperature of the hottest month (b4, layer clipped below $45^{\circ} \mathrm{C}$ temperature), (iv) minimum temperature of the coldest month (b6, layer clipped above $0{ }^{\circ} \mathrm{C}$ temperatures) and (v) precipitation of the wettest month (b13) were obtained from Worldclim website ${ }^{68}$ at a resolution of $0.5 \mathrm{~km}^{2}$. We hypothesized that both species would show a parabolic or peaked response to rainfall and temperature, with a skew toward higher rainfall and a peak at moderate temperatures.

Elevation plays a major role in the distribution of both the megaherbivores as they prefer flatter and less rugged terrain ${ }^{9}$. Ecological studies on rhinoceros indicate preferences for low-lying flat habitats ${ }^{58}$. However, for buffalo, studies have recorded presence up to $1000 \mathrm{~m}$ elevation, so we clipped our modelling extent to elevation below $1000 \mathrm{~m}$. We expected a sharp decline in rhinoceros occurrence with increasing elevation and a similar but shallower decline for buffalo. We used the Digital-Elevation-Model produced by the Shuttle Radar Topography Mission (a joint endeavour by NASA, National Geospatial-intelligence Agency, and German and Italian Agencies) $)^{69}$.

Distance from protected area (PA)

Both megaherbivores suffer poaching and their current populations are largely restricted to PAs ${ }^{35,46}$. We expected species occurrences to decline as distance from PA increased. PA boundaries were taken from the Protected Planet website (https://www.protectedplanet.net/), the Wildlife database cell of the Wildlife Institute of India and the Project Tiger Directorate. The distance of each pixel from PA was calculated using the Euclidean Distance Tool in ESRI ArcMap (10.5.1).

Human footprint

Megaherbivores often conflict with humans due their requirement of vast fertile plains, propensity to crop raiding, damage to human property and lives ${ }^{9}$. Proximity to human settlements also raises the problems of habitat degradation, encroachment, poaching and hunting ${ }^{70}$. We therefore, expected a negative relationship between human footprint index and megaherbivore presence. The Global Human Footprint Index dataset was obtained from Last of the Wild Project ${ }^{71}$, which is the Human Influence Index normalized by biome and realm.

Modelling in maxent. The habitat covariates were exported to ArcMap (10.2) to obtain uniform resolution and concordance with grids of $0.83 \mathrm{~km}^{2}$ across all layers. A correlation matrix for all environmental layers was 
computed in Eris ArcMap (10.5.1) (Table S4). The correlation threshold was kept at $r> \pm 0.70$ to check for redundancy in information. No two correlated variables were used together in a single model.

Maxent operates by developing relationships between known occurrence locations and covariates by comparing them with background locations. Species locations used for training our models were from a restricted part of the modelled space (though all extant species locations were sampled). We therefore used a bias correction file ${ }^{26}$ created using inverse distance weight (IDW) interpolation method in ArcMap (10.5.1) to guide Maxent into picking background locations from space containing occurrence locations ${ }^{54,72}$. This approach would enhance the accuracy of our models by limiting the predictive relationships to be developed from the extent of presence vs background from the same area. We used $80 \%$ of presence locations of each species to train Maxent models and the remaining 20\% were used to test models. The run type was set at Auto with Linear, Hinge, Quadratic and Product functions were selected in Maxent to model relationships between covariates and species occurrence. The maximum number of background points was set at 10,000. Regularization Multiplier was set at default (1) for both species models. We first explored univariate relationships between covariates and species occurrence. Subsequently, based on the univariate responses, we used the covariate combinations that were ecologically meaningful and represented climate, abiotic and biotic habitat and human disturbance to develop more complex models for our species distribution models.

One hundred bootstrap simulations were run for the best model for both species. The median prediction was used to evaluate sites while 95\% upper and lower predictions were used to capture the variability associated with model uncertainty. We report species occurrence probability as spatial maps. To compute patch-areas suitable for reintroduction, we used 'average of maximum training sensitivity plus specificity cumulative threshold' to categorise pixels as suitable habitats ${ }^{23}$. We computed $95 \%$ confidence intervals on suitable habitats using the threshold value. Covariate selection and model evaluation was based on (a) Receiver Operation Characteristic (ROC) curve $\mathrm{C}^{73}$, (b) contribution of covariates individually and in ecologically meaningful combinations to explain the variability of the training and test data sets and (c) Omission/Commission analysis of test dataset (d) True Skill Statistics (TSS) ${ }^{74,75}$ and (e) Akaike Information Criteria (AIC) ${ }^{52,76}$ Species response curves to each covariate were examined and ecologically interpreted ${ }^{77,78}$.

Rhinoceros and buffalo were observed to be at high densities in Kaziranga ( 6 rhinoceros ${ }^{79} / \mathrm{km}^{2}$; and 3.2 buffalos/ $\mathrm{km}^{2}$, https://www.kaziranga-national-park.com/wild-buffaloes.shtml) while Chitwan had lower densities of rhinoceros $\left(1.4 \text { Rhinoceros } / \mathrm{km}^{2}\right)^{80}$. Gorumara NP in West Bengal includes forest, plantation and patchily distributed grasslands carrying a density of about 0.65 rhinoceros in the $\mathrm{PA}^{81,82}$. These differences were likely due to difference in productivity and habitat availability in these PAs; with Kaziranga being more productive and the entire PA being favourable habitat while Chitwan (constituted by Terai, Bhabhar, and Churia hills) and Gorumara having only parts of their habitat as favourable. We report crude densities for these PA but use a conservative estimate of one individual $\mathrm{km}^{-2}$ (and $2 \mathrm{~km}^{-2}$ for Orang TR which has habitat similar to Kaziranga) as the potential ecological density of both species for reintroduction sites. Achievable population sizes were computed by multiplying only the suitable habitat area by this density.

Population habitat viability analysis (PHVA). We assessed the viability of potential reintroduced and extant populations using PHVA in Vortex $9.93^{21}$. PHVA allows assessment of persistence of a population over a specified timespan incorporating the stochastic nature of demographic parameters, carrying capacity, environmental fluctuations, genetic variability, and deterministic impacts such as poaching ${ }^{83}$. Information on demographic parameters for rhinoceros was obtained from previous studies in Chitwan ${ }^{39,58,67}$. Demographic parameters for the buffalo were also obtained from literature ${ }^{16,84}$ (Table S5). We modelled populations with carrying capacities $(\mathrm{K})$ ranging between 10-150 for rhinoceros and 20-500 for buffalos to reflect habitat patch sizes estimated through Maxent. Each population was subjected to scenarios with varying sizes of initial population, levels of supplementation, poaching and mortality caused by catastrophe (floods/diseases). Based on population sizes and potential movement between Kaziranga, Orang and Laukhowa-Burachapori, a metapopulation of rhinoceros was modelled. This model provided information on the role of movement across the landscape upon viability of individual populations and the metapopulation. Five hundred simulations for each scenario were run for 100 years. Extinction probability, stochastic growth rate, population size, persistence time and the level of heterozygosity were used as evaluation criteria $^{39}$ for prioritizing potential sites.

Selection criteria for reintroduction sites and recommendations. We considered habitat clusters of combined minimum size $>50 \mathrm{~km}^{2}$ to include only those sites which could hold significant populations by themselves or as a metapopulation. We assessed the identified potential reintroduction sites on: (a) habitat quality and size, (b) potential to sustain reintroduced populations over 100 years, (c) legal status and level of protection available/possible, (d) level of anthropogenic stressors and likely conflict with communities.

Received: 10 August 2020; Accepted: 30 October 2020

Published online: 24 February 2021

\section{References}

1. Ceballos, G. et al. Accelerated modern human-induced species losses: Entering the sixth mass extinction. Sci. Adv. 1, e1400253 (2015).

2. Cardillo, M. et al. Human population density and extinction risk in the world's carnivores. PLoS Biol. 2, 909-914 (2004).

3. Pimm, S. L. et al. Can we defy nature's end ?. Science 293, 2207-2208 (2001). 
4. Ripple, W. J. et al. Bushmeat hunting and extinction risk to the world's mammals. R. Soc. Open Sci. 3, 160498 (2016).

5. Chapron, G. et al. Recovery of large carnivores in Europe's modern human-dominated landscapes. Science 346, 1517-1519 (2014).

6. Owen-Smith, N. Pleistocene extinctions: the pivotal role of megaherbivores. Paleobiology 13, 351-362 (1987).

7. Pradhan, N. M. B. \& Wegge, P. Dry season habitat selection by a recolonizing population of Asian elephants Elephas maximus in lowland Nepal. Acta Theriol. (Warsz) 52, 205-214 (2007).

8. Hayward, M. W. et al. The reintroduction of large carnivores to the Eastern Cape South Africa: an assessment. Oryx 41(205), 214 (2007).

9. Owen-Smith, N. Megaherbivores: the influence of very large body size on ecology. Trends Ecol. Evol. https://doi. org/10.1111/j.1523-1739.1989.tb00246.x (1989).

10. Karki, J. B., Jhala, Y. V. \& Khanna, P. P. Grazing lawns in Terai Grasslands, Royal Bardia National Park, Nepal1. Biotropica 32, 423-429 (2000).

11. McNaughton, S. J. Serengeti migratory wildebeest: facilitation of energy flow by grazing. Science 191, 92-94 (1976).

12. Skarpe, C. et al. The return of the giants: ecological effects of an increasing elephant population. Ambio 33, 276-282 (2004).

13. Foose, T. J. \& Van Strien, N. Asian Rhinos-Status Survey and Conservation Action Plan. Vol. 32 (1997).

14. Subedi, N. et al. Population status, structure and distribution of the greater one-horned rhinoceros Rhinoceros unicornis in Nepal. Oryx 47, 352-360 (2013).

15. Talukdar, B. R. R. C. Asian Rhino specialist group report. Pachyderm 53, 25-27 (2013).

16. Hedges, S., Sagar Baral, H., Timmins, R. \& Duckworth, J. Bubalus arnee. IUCNRed List Threat. Species 2008 (2008).

17. Leader-Williams, N. Fate riding on their horns-and genes?. ORYX 47, 311-312 (2013).

18. Amin, R., Thomas, K., Emslie, R. H., Foose, T. J. \& VanStrien, N. An overview of the conservation status of and threats to rhinoceros species in the wild. Int. Zoo Yearb. 40, 96-117 (2006).

19. IUCN Red List Categories and Criteria, Version 3.1, second edition | IUCN Library System. IUCN (2012).

20. Phillips, S. J. \& Dudík, M. Modeling of species distributions with Maxent: new extensions and a comprehensive evaluation. Ecography 31, 161-175 (2008).

21. Lacy, R. C. Vortex: A computer simulation model for population viability analysis. Wildl. Res. 20, 1-13 (1993).

22. IUCN/SSC. Guidelines for Reintroductions and Other Conservation Translocations. Version 1.0. Gland, Switzerland: IUCN Species Survival Commission. Ecologial Applications (2013).

23. Guillera-Arroita, G. et al. Is my species distribution model fit for purpose? Matching data and models to applications. Glob. Ecol. Biogeogr. 24, 276-292 (2015).

24. Elith, J. \& Leathwick, J. R. Species distribution models: ecological explanation and prediction across space and time. Annu. Rev. Ecol. Evol. Syst. 40, 677-697 (2009).

25. Veloz, S. D. Spatially autocorrelated sampling falsely inflates measures of accuracy for presence-only niche models. J. Biogeogr. 36, 2290-2299 (2009).

26. Kramer-Schadt, S. et al. The importance of correcting for sampling bias in MaxEnt species distribution models. Divers. Distrib. 19, 1366-1379 (2013).

27. Jhala, Y., Qureshi, Q. \& Gopal, R. Status of Tigers, Copredators and Prey in India, 2014 (2015).

28. Graham-Rowe, D. Biodiversity: endangered and in demand. Nature 480, S101-S103 (2011).

29. Frankham, R. Genetic considerations in reintroduction programmes for top-order, terrestrial predators. In Reintroduction of TopOrder Predators 371-387 (Wiley-Blackwell, 2009). https://doi.org/10.1002/9781444312034.ch17

30. Martin, E., Kumar Talukdar, B. \& Vigne, L. Rhino poaching in Assam: challenges and opportunities. Pachyderm (2009).

31. Rawat, G. S., Goyal, S. P. \& Johnsingh, A. J. T. Ecological observations on the grasslands of Corbett Tiger Reserve India. Indian Forester 123(958), 963 (1997).

32. Dinerstein, E. \& Schaller, G. The Return of the Unicorns: Natural History and Conservation of Greater-One Horned Rhinoceros (2003). https://doi.org/10.7312/dine08450.

33. N Subedi 2012 Effect of Mikania micrantha on thrdemography, Habitat use, and Nutrition of Greater one horned rhinoceros in Chitwan National Park Dr. Philos Thesis

34. Mathur, V.B., Gopal, R., Yadav, S.P., P. R. S. Management Effectiveness Evaluation (MEE) of Tiger Reserves in India: Process and Outcomes 97 (2011).

35. Amin, R., Thomas, K., Emslie, R. H., Foose, T. J. \& Van Strien, N. V. An overview of the conservation status of and threats to rhinoceros species in the wild. Int. Zoo Yearb. 40, 96-117 (2006).

36. Singh, S. P., Sharma, A. \& Talukdar, B. K. Translocation of Rhinos within Assam : a successful third round of the second phase of translocations under Indian Rhino Vision (IRV) 2020, 1-6 (2012).

37. Jhala, Y. ., Qureshi, Q., Gopal, R. \& Sinha, P. . Status of the Tigers, Co-predators, and Prey in India, 2010. (2011).

38. Rai, S. After 250 years, rhinos set to make comeback in west UP|Meerut News-Times of India. Times of India (2016).

39. Subedi, N., Lamichhane, B. R., Amin, R., Jnawali, S. R. \& Jhala, Y. V. Demography and viability of the largest population of greater one-horned rhinoceros in Nepal. Glob. Ecol. Conserv. 12, 241-252 (2017).

40. IUCN Standards and Petitions Committee. IUCN Standards and Petitions Committee. Stand. Petitions Comm. 1, 1-60 (2019).

41. Cockrill, W. R. The water buffalo: a review. Br. Vet. J. 137, 8-10 (1981).

42. Kumar, S. et al. Mitochondrial DNA analyses of Indian water buffalo support a distinct genetic origin of river and swamp buffalo. Anim. Genet. 38, 227-232 (2007).

43. Aiyadurai, A. Wildlife hunting and conservation in Northeast India-a need for an interdisciplinary understanding.pdf. Int. J. Gall. Conserv. 2, 61-73 (2011).

44. Jhala, H. Y., Pokheral, C. P. \& Subedi, N. Well being and conservation awareness of communities around Chitwan National Park, Nepal|Jhala|Indian Forester. Indian For. 145, 114-120 (2019).

45. Heinen, J. T. \& Kandel, R. Threats to a small population: a census and conservation recommendations for wild buffalo Bubalus arnee in Nepal. Oryx 40, 324-330 (2006).

46. Choudhury, A. The decline of the wild water buffalo in north-east India. Oryx 28, 70-73 (1994).

47. Magioncalda, W. A modern insurgency: India’s evolving naxalite problem. S. Asia Monitor 140 (2010).

48. Melles, S. J., Fortin, M.-J., Lindsay, K. \& Badzinki, D. Expanding northward: influence of climate change, forest connectivity, and population processes on a threatened species' range shift. Glob. Chang. Biol. 17, 17-31 (2011).

49. Challender, D. W. S. \& MacMillan, D. C. Poaching is more than an enforcement problem. Conserv. Lett. 7, 484-494 (2014).

50. Hirzel, A. H., Hausser, J., Chessel, D. \& Perrin, N. Ecological-niche factor analysis: How to compute habitat-suitability maps without absence data?. Ecology 83, 2027-2036 (2002).

51. Stockman, A. K., Beamer, D. A. \& Bond, J. E. An evaluation of a GARP model as an approach to predicting the spatial distribution of non-vagile invertebrate species. Divers. Distrib. 12, 81-89 (2006).

52. Merow, C., Smith, M. J. \& Silander, J. A. A practical guide to MaxEnt for modeling species' distributions: what it does, and why inputs and settings matter. Ecography (Cop.) 36, 1058-1069 (2013).

53. Phillips, S. J. \& Dudi, M. Modeling of species distributions with Maxent : new extensions and a comprehensive evaluation. Ecograohy 31, 161-175 (2008)

54. Elith, J. et al. A statistical explanation of MaxEnt for ecologists. Divers. Distrib. 17, $43-57$ (2011). 
55. Phillips, S. J., Anderson, R. P., Dudík, M., Schapire, R. E. \& Blair, M. E. Opening the black box: an open-source release of Maxent. Ecography (Cop.) 40, 887-893 (2017).

56. Congalton, R. G. A review of assessing the accuracy of classifications of remotely sensed data. Remote Sens. Environ. 37, 35-46 (1991).

57. Roy, D. P. et al. Landsat-8: Science and product vision for terrestrial global change research. Remote Sens. Environ. 145, 154-172 (2014).

58. Dinerstein, E. \& Price, L. Demography and habitat use by greater one-horned rhinoceros in Nepal. J. Wildl. Manage. 55, 401 (1991). 59. Bontemps, S. et al. GLOBCOVER 2009 Products Description and Validation Report.

60. Barsi, J., Lee, K., Kvaran, G., Markham, B. \& Pedelty, J. The spectral response of the landsat-8 operational land imager. Remote Sens. 6, 10232-10251 (2014).

61. Laurie, A. Behavioural ecology of the Greater one-horned rhinoceros (Rhinoceros unicornis). J. Zool. 196, 307-341 (2009).

62. Dnerstein, E. \& Price, L. Demography and habitat use by greater one-horned rhinoceros in Nepal author(s): Eric Dinerstein and Lori Price Source. J. Wildl. Manage. 55, 401-411 (1991).

63. Pettorelli, N. et al. The Normalized Difference Vegetation Index (NDVI): unforeseen successes in animal ecology. Climate Res. 46, $15-27$ (2011).

64. Raffini, F. et al. From nucleotides to satellite imagery: approaches to identify and manage the invasive pathogen Xylella fastidiosa and its insect vectors in Europe. Sustainability 12, 4508 (2020).

65. Norberg, A. et al. A comprehensive evaluation of predictive performance of 33 species distribution models at species and community levels. Ecol. Monogr. https://doi.org/10.1002/ecm.1370 (2019).

66. Peterson, A. T. \& Nakazawa, Y. Environmental data sets matter in ecological niche modelling: an example with Solenopsis invicta and Solenopsis richteri. Glob. Ecol. Biogeogr. https://doi.org/10.1111/j.1466-8238.2007.00347.x (2007).

67. Laurie, A. Behavioural ecology of the greater one-horned rhinoceros Rhinoceros unicornis. J. Zool. 196, 307-341 (1982).

68. Kriticos, D. J. et al. CliMond: Global high-resolution historical and future scenario climate surfaces for bioclimatic modelling. Methods Ecol. Evol. 3, 53-64 (2012).

69. Rodríguez, E. et al. An Assessment of the SRTM Topographic Products.

70. Watson, J. E. M., Whittaker, R. J. \& Dawson, T. P. Habitat structure and proximity to forest edge affect the abundance and distribution of forest-dependent birds in tropical coastal forests of southeastern Madagascar. Biol. Conserv. 120, 311-327 (2004).

71. Society WC. Last of the Wild Project, Version 2, 2005 (LWP-2): Global Human Influence Index (HII) Dataset (Geographic) Society. Conserv Wildl https://doi.org/10.7927/H4BP00QC (2005).

72. Phillips, S. J. et al. Sample selection bias and presence-only distribution models: Implications for background and pseudo-absence data. Ecol. Appl. 19, 181-197 (2009).

73. Jiménez-Valverde, A. Insights into the area under the receiver operating characteristic curve (AUC) as a discrimination measure in species distribution modelling. Glob. Ecol. Biogeogr. 21, 498-507 (2011).

74. Allouche, O., Tsoar, A. \& Kadmon, R. Assessing the accuracy of species distribution models: prevalence, kappa and the true skill statistic (TSS). J. Appl. Ecol. 43, 1223-1232 (2006).

75. Shabani, F., Kumar, L. \& Ahmadi, M. Assessing accuracy methods of species distribution models: AUC, specificity, sensitivity and the true skill statistic. 18, (2018).

76. Warren, D. L. \& Seifert, S. Ecological niche modeling in Maxent: the importance of model complexity and the performance of model selection criteria. Ecol. Soc. Am. 21, 335-342 (2011).

77. Wiltshire, K. H. \& Tanner, J. E. Comparing maximum entropy modelling methods to inform aquaculture site selection for novel seaweed species. Ecol. Modell. 429, 109071 (2020).

78. Smeraldo, S. et al. Modelling risks posed by wind turbines and power lines to soaring birds: the black stork (Ciconia nigra) in Italy as a case study. Biodivers. Conserv. 29, 1959-1976 (2020).

79. Puri, K. \& Joshi, R. A case study of greater one horned rhinoceros (Rhinoceros unicornis) in Kaziranga National Park of Assam India. NeBIO 9, 307-309 (2018).

80. DNPWC 2015. Rhino Count Report 2015 DNPWC.

81. Mukherjee, T., Sharma, L. K., Saha, G. K., Thakur, M. \& Chandra, K. Past, present and future: combining habitat suitability and future landcover simulation for long-term conservation management of Indian rhino. Sci. Rep. https://doi.org/10.1038/s4159 8-020-57547-0 (2020).

82. CCF West Bengal. Estimation of Indian Rhinoceros (Rhinoceros unicornis) 2019 West Bengal (2019)

83. Boyce, M. Population viability analysis: adaptive management for threatened and endangered species. 226-238 (1997).

84. Khatri, T. B., Shah, D. N. \& Mishra, N. Wild Water Buffalo Bubalus arnee in Koshi Tappu Wildlife Reserve, Nepal: status, population and conservation importance. J. Threat. Taxa 04, 3294-3301 (2012).

85. Phillips, S., Anderson, R., Dudík, M., Schapire, R. \& Blair, M. Opening the black box: an open-source release of Maxent. Ecography 40, 887-893 (2017).

\section{Acknowledgements}

We would like to thank V. B. Mathur, Director Wildlife Institute of India and the G. S. Rawat Dean for their support. We thank the University of Kent and Durrell Institute of Conservation and Ecology for providing funding. Ninad Mungi and Swati Saini are acknowledged for their help with GIS. We thank Protected Area and State officials for providing insights on management challenges. We thank P. J. Deka for information on Swamp Buffalo. We thank N Subedi from the National Trust Nature Conservation, Nepal, for sharing data on rhino and buffalo locations. R. Y. Jhala is especially thanked for her support.

\section{Author contributions}

H.Y.J.: conceived the study, collected primary data in the field and conducted interviews, analysed the data, and wrote the manuscript. Q.Q.: supervised field data collection and advised on analysis. Y.V.J.: assisted in study design, field data collection, analysis, interpretation, and writing the manuscript. S.A.B.: supervised writing M.Sc. thesis, sourced key historical species literature, provided resources, and reviewed the manuscript.

\section{Competing interests}

The authors declare no competing interests.

\section{Additional information}

Supplementary Information The online version contains supplementary material available at https://doi. org/10.1038/s41598-021-83174-4. 
Correspondence and requests for materials should be addressed to H.Y.J. or S.A.B.

Reprints and permissions information is available at www.nature.com/reprints.

Publisher's note Springer Nature remains neutral with regard to jurisdictional claims in published maps and institutional affiliations.

(c) (i) Open Access This article is licensed under a Creative Commons Attribution 4.0 International cc) License, which permits use, sharing, adaptation, distribution and reproduction in any medium or format, as long as you give appropriate credit to the original author(s) and the source, provide a link to the Creative Commons licence, and indicate if changes were made. The images or other third party material in this article are included in the article's Creative Commons licence, unless indicated otherwise in a credit line to the material. If material is not included in the article's Creative Commons licence and your intended use is not permitted by statutory regulation or exceeds the permitted use, you will need to obtain permission directly from the copyright holder. To view a copy of this licence, visit http://creativecommons.org/licenses/by/4.0/.

(C) The Author(s) 2021 